\title{
Automorphic vector bundles on the stack of $G$-zips
}

\author{
Naoki Imai ${ }^{1}$ and Jean-Stefan Koskivirta ${ }^{2}$ \\ ${ }^{1}$ Graduate School of Mathematical Sciences, The University of Tokyo, 3-8-1 Komaba, Meguro-ku, Tokyo, 153-8914, Japan; \\ E-mail: naoki@ms.u-tokyo.ac.jp. \\ ${ }^{2}$ Department of Mathematics, Faculty of Science, Saitama University, 255 Shimo-Okubo, Sakura-ku, Saitama City, Saitama \\ 338-8570, Japan; E-mail: jeanstefan.koskivirta@gmail.com.
}

Received: 9 September 2020; Accepted: 1 March 2021

2020 Mathematics Subject Classification: Primary - 14G35; Secondary - 20G40

\begin{abstract}
For a connected reductive group $G$ over a finite field, we study automorphic vector bundles on the stack of $G$-zips. In particular, we give a formula in the general case for the space of global sections of an automorphic vector bundle in terms of the Brylinski-Kostant filtration. Moreover, we give an equivalence of categories between the category of automorphic vector bundles on the stack of $G$-zips and a category of admissible modules with actions of a 0-dimensional algebraic subgroup a Levi subgroup and monodromy operators.
\end{abstract}

\section{Introduction}

The stack of $G$-zips was introduced by Pink-Wedhorn-Ziegler [PWZ11] and [PWZ15] based on the notion of F-zip defined in the work of Moonen-Wedhorn ([MW04]). In this article, we investigate vector bundles on the stack of $G$-zips. Let $G$ be a connected reductive group over a finite field $\mathbb{F}_{q}$ and let $k$ denote an algebraic closure of $\mathbb{F}_{q}$. For a cocharacter $\mu: \mathbb{G}_{\mathrm{m}, k} \rightarrow G_{k}$, Pink-Wedhorn-Ziegler have defined a smooth finite stack $G$-Zip ${ }^{\mu}$ over $k$, called the stack of $G$-zips of type $\mu$. Many authors have shown that it is a useful tool to study the geometry of Shimura varieties in characteristic $p$. For example, let $\operatorname{Sh}(\mathbf{G}, \mathbf{X})_{K}$ be a Shimura variety of Hodge type over a number field $\mathbf{E}$ with good reduction at a prime $p$. Kisin [Kis10] and Vasiu [Vas99] have constructed an integral model $\mathcal{S}_{K}$ over $\mathcal{O}_{\mathbf{E}_{v}}$ at all places $v \mid p$ in $\mathbf{E}$. Denote by $S_{K}$ the geometric special fibre of $\mathcal{S}_{K}$ and by $G$ the special fibre over $\mathbb{F}_{p}$ of $\mathbf{G}$ (in the context of Shimura varieties, we take $q=p$ ). Let $\mu$ be the cocharacter attached naturally to $\mathbf{X}$. Then Zhang [Zha18] has shown that there exists a smooth morphism of stacks $\zeta: S_{K} \rightarrow G$-Zip ${ }^{\mu}$, which is also surjective. The second author and Wedhorn have used the stack $G$-Zip ${ }^{\mu}$ to construct $\mu$-ordinary Hasse invariants in [KW18], and this result was later generalised to all Ekedahl-Oort strata with Goldring [GK19a].

In [Kos19], the second author studied the space of global sections of the family of vector bundles $\left(\mathcal{V}_{I}(\lambda)\right)_{\lambda \in X^{*}(T)}$. To explain what these vector bundles are, first recall that the cocharacter $\mu$ yields a parabolic subgroup $P \subset G_{k}$ as well as a Levi subgroup $L \subset P$, which is equal to the centraliser of $\mu$ (see Subsection 2.2.2 for details). Then for any algebraic $P$-representation $(V, \rho)$ over $k$, there is a naturally attached vector bundle $\mathcal{V}(\rho)$ of $\operatorname{rank} \operatorname{dim}(V)$ on $G$-Zip ${ }^{\mu}$ modelled on $(V, \rho)$ (see Subsection 2.4). We call $\mathcal{V}(\rho)$ an automorphic vector bundle on $G$-Zip ${ }^{\mu}$ (cf. [Mil90, Chapter III, §2]).

The vector bundle $\mathcal{V}_{I}(\lambda)$ (for $\lambda \in X^{*}(T)$ a character of a maximal torus $T \subset G$ ) is by definition the vector bundle attached to the $P$-representation $V_{I}(\lambda)=\operatorname{Ind}_{B}^{P}(\lambda)$, where $B \subset P$ is a Borel subgroup (containing $T$ and appropriately chosen), Ind denotes induction and $I$ denotes the set of simple roots 
of $L$. For a $k$-algebraic group $H$, we write $\operatorname{Rep}(H)$ for the category of finite-dimensional algebraic representations of $H$ over $k$. The natural projection $P \rightarrow L$ modulo the unipotent radical induces a fully faithful functor $\operatorname{Rep}(L) \rightarrow \operatorname{Rep}(P)$. In particular, all representations of the form $V_{I}(\lambda)$ lie in the full subcategory $\operatorname{Rep}(L)$. In the case when $G$ is split over $\mathbb{F}_{p}$, we showed in a previous work [Kos 19 , Theorem 1] that $H^{0}\left(G\right.$-Zip $\left.{ }^{\mu}, V_{I}(\lambda)\right)$ can be expressed as

$$
H^{0}\left(G-\operatorname{Zip}^{\mu}, \mathcal{V}_{I}(\lambda)\right)=V_{I}(\lambda)^{L\left(\mathbb{F}_{p}\right)} \cap V_{I}(\lambda)_{\leq 0},
$$

where $V_{I}(\lambda)^{L\left(\mathbb{F}_{p}\right)}$ denotes the $L\left(\mathbb{F}_{p}\right)$-invariant subspace of $V_{I}(\lambda)$ and $V_{I}(\lambda)_{\leq 0} \subset V_{I}(\lambda)$ is defined as follows: It is the direct sum of the $T$-weight spaces $V_{I}(\lambda)_{v}$ for the weights $v$ satisfying $\left\langle v, \alpha^{\vee}\right\rangle \leq 0$ for any simple root $\alpha$ outside of $L$.

In this article, we vastly generalise the formula (1.0.1) to the most general case. We do not assume that $G$ is split over $\mathbb{F}_{q}$ and, more important, we consider arbitrary representations in the larger category $\operatorname{Rep}(P)$ as opposed to the subcategory $\operatorname{Rep}(L)$. In the context of Shimura varieties, there are many interesting vector bundles other than the family $\left(V_{I}(\lambda)\right)_{\lambda}$, which may not always arise from representations in $\operatorname{Rep}(L)$. For example, in [Urb14], nearly holomorphic modular forms of weight $k$ and order $\leq r$ are defined as sections of the vector bundle $\omega^{\otimes(k-r)} \otimes \operatorname{Sym}^{r}\left(\mathcal{H}_{\mathrm{dR}}^{1}\right)$ on the modular curve $X(N)$ for some level $N \geq 1$. Here, $\mathcal{H}_{\mathrm{dR}}^{1}$ is the sheaf of relative de Rham cohomology of the universal elliptic curve $\mathscr{E} \rightarrow X(N)$, and $0 \subset \omega \subset \mathcal{H}_{\mathrm{dR}}^{1}$ is the usual Hodge filtration. In this context, the group $G$ is $\mathrm{GL}_{2}, P=B$ is a Borel subgroup of $G$. The vector bundle $\mathcal{H}_{\mathrm{dR}}^{1}$ is attached to the dual of the standard representation of $\mathrm{GL}_{2}$ (viewed by restriction as a representation of $P$ ). Similarly, $\operatorname{Sym}^{r}\left(\mathcal{H}_{\mathrm{dR}}^{1}\right)$ is attached to the $r$ th symmetric power of that representation. More generally, on the Siegel-type Shimura variety $\mathscr{A}_{g}$ (which parametrise principally polarised abelian varieties of rank $g$ ), the universal abelian scheme yields a rank $2 g$ vector bundle $\mathcal{H}_{\mathrm{dR}}^{1}$ on $\mathscr{A}_{g}$. One can extend the definition of $\mathcal{H}_{\mathrm{dR}}^{1}$ to Hodge-type Shimura varieties after choosing a Siegel embedding. Furthermore, it extends to a vector bundle on the integral model $\delta_{K}$ of Kisin and Vasiu. This example shows that it is desirable to also understand vector bundles that arise from general representations of $P$. In this article, we determine the space $H^{0}\left(G\right.$-Zip $\left.{ }^{\mu}, \mathcal{V}(\rho)\right)$ for any cocharacter datum $(G, \mu)$ (for the definition of cocharacter datum, see Subsection 2.2.2) and for any representation $(V, \rho) \in \operatorname{Rep}(P)$. By Zhang's smooth surjective map $\zeta: S_{K} \rightarrow G$-Zip ${ }^{\mu}$, this determines a natural Hecke-equivariant subspace

$$
H^{0}\left(G-\text { Zip }^{\mu}, \mathcal{V}(\rho)\right) \underset{\zeta^{*}}{\longrightarrow} H^{0}\left(S_{K}, \mathcal{V}(\rho)\right) \text {. }
$$

In particular, we obtain Hecke-equivariant sections of $\mathcal{V}(\rho)$ on $S_{K}$. Furthermore, we can potentially study sections on Ekedahl-Oort strata by the same method, as demonstrated in [GK19a]. Another motivation for describing sections on $G$-Zip ${ }^{\mu}$ is that we would like to determine which weights $\lambda$ admit nonzero automorphic forms. Specifically, let $C_{K}$ denote the set of $\lambda \in X^{*}(T)$ such that $H^{0}\left(S_{K}, \mathcal{V}_{I}(\lambda)\right) \neq 0$. Similarly, let $C_{\text {zip }}$ be the set of $\lambda$ such that $H^{0}\left(G-\operatorname{Zip}^{\mu}, \mathcal{V}_{I}(\lambda)\right) \neq 0$ (one can show that they are cones in $X^{*}(T)$ ). The inclusion (1.0.2) shows that $C_{\text {zip }} \subset C_{K}$. Denote by $(-)_{\mathbb{Q}>0}$ the generated $\mathbb{Q}_{>0}$-cones. Then one can see [Kos19, Corollary 1.5.3] that $C_{K, \mathbb{Q}_{>0}}$ is independent of $K$, and we conjecture [GK18, Conjecture 2.1.6] that it coincides with $C_{\text {zip, } \mathbb{Q}_{>0}}$. Goldring and the second author proved this conjecture in some case in [GK18, Theorem D].

We show that the space $H^{0}\left(G-Z_{i p}, \mathcal{V}(\rho)\right)$ is given by the intersection of the $L_{\varphi}$-invariants of $V$ with a generalised Brylinski-Kostant filtration (where $L_{\varphi} \subset L$ is a certain 0-dimensional group, see (3.2.1)). For the general statement, see Theorem 3.4.1. For the sake of brevity, we give a simplified statement in this introduction. Assume here that $P$ is defined over $\mathbb{F}_{q}$ (in this case, $L_{\varphi}=L\left(\mathbb{F}_{q}\right)$ ). Let $\wp^{*}: X^{*}(T)_{\mathbb{R}} \rightarrow X^{*}(T)_{\mathbb{R}}$ be the map induced by the Lang torsor $\wp: T \rightarrow T ; g \mapsto g \varphi(g)^{-1}$, where $\varphi: G \rightarrow G$ denotes the $q$ th power Frobenius homomorphism. Let $V=\bigoplus_{v} V_{v}$ be the weight decomposition of $V$. For $\chi \in X^{*}(T)_{\mathbb{R}}$, let Fil ${ }_{\chi}^{P} V_{v}$ be the Brylinski-Kostant filtration of $V_{v}$ (see (3.4.2)). 
Theorem 1 (Corollary 3.4.2). Assume that $P$ is defined over $\mathbb{F}_{q}$. For any $(V, \rho) \in \operatorname{Rep}(P)$, we have

$$
H^{0}\left(G-\mathrm{Zip}^{\mu}, \mathcal{V}(\rho)\right)=V^{L\left(\mathbb{F}_{q}\right)} \cap \bigoplus_{v \in X^{*}(T)} \operatorname{Fil}_{\wp^{*-1}(v)}^{P} V_{\nu}
$$

In the more simple case of [Kos19], the space $V_{I}(\lambda)_{\leq 0}$ appearing in equation (1.0.1) is a sum of weight spaces of $V$. In the general case, $H^{0}\left(G-Z i p^{\mu}, \mathcal{V}(\rho)\right)$ cannot be written as an intersection of $V^{L\left(\mathbb{F}_{q}\right)}$ with a sum of weight spaces of $V$ (see Examples 4.3.2 for a counterexample). We include examples of concrete computations of the space $H^{0}\left(G\right.$-Zip $\left.{ }^{\mu}, \mathcal{V}(\rho)\right)$ in Section 6.

Our second result concerns the category $\mathfrak{B B}\left(G\right.$-Zip $\left.{ }^{\mu}\right)$ of vector bundles on $G$-Zip ${ }^{\mu}$. As explained above, there is a natural functor $\mathcal{V}: \operatorname{Rep}(P) \rightarrow \mathfrak{B B}\left(G\right.$-Zip $\left.{ }^{\mu}\right)$. Denote by $\mathfrak{B B}_{P}\left(G\right.$-Zip $\left.{ }^{\mu}\right)$ the full subcategory that is equal to the essential image of $\mathcal{V}$. We give an explicit description of the category $\mathfrak{B} \mathfrak{B}_{P}\left(G\right.$-Zip $\left.{ }^{\mu}\right)$ of automorphic vector bundles. We define the category of $L_{\varphi}$-modules with $\Delta^{P}$ monodromy (see Definition 5.2.2). Its objects are $L_{\varphi}$-modules $W$ endowed with a set of monodromy operators indexed by $\Delta^{P}$ (where $\Delta^{P}$ denotes the set of simple roots outside the parabolic $P$ ). There is a natural functor $F_{\mathrm{MN}}$ : $\operatorname{Rep}(P) \rightarrow L_{\varphi}-\mathrm{MN}_{\Delta^{P}}$ (see (5.2.1)). An $L_{\varphi}$-module with $\Delta^{P}$-monodromy is called admissible if it lies in the essential image of $F_{\mathrm{MN}}$. The category of admissible $L_{\varphi}$-modules $\Delta^{P}$-monodromy is denoted by $L_{\varphi}-\mathrm{MN}_{\Delta^{P}}^{\mathrm{adm}}$.

Theorem 2 (Theorem 5.1.5). The functor $\mathcal{V}: \operatorname{Rep}(P) \rightarrow \mathfrak{B B}\left(G\right.$-Zip $\left.{ }^{\mu}\right)$ factors through the functor $F_{\mathrm{MN}}: \operatorname{Rep}(P) \rightarrow L_{\varphi}-\mathrm{MN}_{\Delta^{P}}^{\text {adm }}$ and induces an equivalence of categories

$$
L_{\varphi}-\mathrm{MN}_{\Delta^{P}}^{\mathrm{adm}} \longrightarrow \mathfrak{B} \mathfrak{B}_{P}\left(G-\mathrm{Zip}^{\mu}\right) .
$$

In particular, we deduce the following. Let $S_{K}$ denote again the good reduction special fibre of a Hodge-type Shimura variety. Similarly, there is a natural functor $\operatorname{Rep}(P) \rightarrow \mathfrak{B B}\left(S_{K}\right)$, where $\mathfrak{B B}\left(S_{K}\right)$ denotes the category of vector bundles on $S_{K}$. Write again $\mathfrak{B} \mathfrak{B}_{P}\left(S_{K}\right)$ for the essential image of $\operatorname{Rep}(P)$. In this context, we have the following.

Corollary 3 (Corollary 5.1.6). The functor $\mathcal{V}: \operatorname{Rep}(P) \rightarrow \mathfrak{B} \mathfrak{B}_{P}\left(S_{K}\right)$ factors as

$$
\operatorname{Rep}(P) \stackrel{F_{\mathrm{MN}}}{\longrightarrow} L_{\varphi^{-}}-\mathrm{MN}_{\Delta^{P}}^{\mathrm{adm}} \stackrel{\zeta^{*}}{\longrightarrow} \mathfrak{B} \mathfrak{B}_{P}\left(S_{K}\right)
$$

The results of this article will be used in the follow-up articles [IK21] and [GIK21], where we study partial Hasse invariants for Shimura varieties of Hodge type.

\section{Vector bundles on the stack of $G$-zips}

\subsection{Notation}

Throughout the article, $p$ is a prime number, $q$ is a power of $p$ and $\mathbb{F}_{q}$ is the finite field with $q$ elements. We write $k=\overline{\mathbb{F}}_{q}$ for an algebraic closure of $\mathbb{F}_{q}$. Write $\sigma \in \operatorname{Gal}\left(k / \mathbb{F}_{q}\right)$ for the $q$ th power Frobenius. For a $k$-scheme $X$ and $m \in \mathbb{Z}$, we write $X^{\left(q^{m}\right)}$ for the base change of $X$ by $\sigma^{m}$ and $\varphi: X^{\left(q^{m}\right)} \rightarrow X^{\left(q^{m+1}\right)}$ for the relative $q$ th power Frobenius morphism. For an algebraic representation $(V, \rho)$ of an algebraic group $H$ over $k$, let $\left(V^{(q)}, \rho^{(q)}\right)$ denote the representation $\rho \circ \varphi: H^{\left(q^{-1}\right)} \rightarrow H \rightarrow \operatorname{GL}(V)$.

The notation $G$ will denote a connected reductive group over $\mathbb{F}_{q}$. We will always write $(B, T)$ for a Borel pair defined over $\mathbb{F}_{q}$; that is, $T \subset B \subset G_{k}$ are a maximal torus and a Borel subgroup defined over $\mathbb{F}_{q}$. Let $B^{+}$be the Borel subgroup of $G_{k}$ opposite to $B$ with respect to $T$ (i.e., the unique Borel subgroup of $G$ such that $B^{+} \cap B=T$ ). We will use the following notations:

- As usual, $X^{*}(T)$ (respectively $X_{*}(T)$ ) denotes the group of characters (respectively cocharacters) of $T$. The group $\operatorname{Gal}\left(k / \mathbb{F}_{q}\right)$ acts naturally on these groups. Let $W=W\left(G_{k}, T\right)$ be the Weyl group of 
$G_{k}$. Similarly, $\operatorname{Gal}\left(k / \mathbb{F}_{q}\right)$ acts on $W$. Furthermore, the actions of $\operatorname{Gal}\left(k / \mathbb{F}_{q}\right)$ and $W$ on $X^{*}(T)$ and $X_{*}(T)$ are compatible in a natural sense.

- $\Phi \subset X^{*}(T)$ : the set of $T$-roots of $G$.

- $\Phi_{+} \subset \Phi$ : the system of positive roots with respect to $B^{+}$(i.e., $\alpha \in \Phi_{+}$when the $\alpha$-root group $U_{\alpha}$ is contained in $B^{+}$). This convention may differ from other authors. We use it to match the conventions of [Jan03, Chapter II, §1.8] and previous publications [GK19a], [Kos19].

$\circ \Delta \subset \Phi_{+}$: the set of simple roots.

○ For $\alpha \in \Phi$, let $s_{\alpha} \in W$ be the corresponding reflection. The system $\left(W,\left\{s_{\alpha}\right\}_{\alpha \in \Delta}\right)$ is a Coxeter system. Write $\ell: W \rightarrow \mathbb{N}$ for the length function. Hence, $\ell\left(s_{\alpha}\right)=1$ for all $\alpha \in \Phi$. Let $w_{0}$ denote the longest element of $W$.

○ For a subset $K \subset \Delta$, let $W_{K}$ denote the subgroup of $W$ generated by $\left\{s_{\alpha}\right\}_{\alpha \in K}$. Write $w_{0, K}$ for the longest element in $W_{K}$.

- Let ${ }^{K} W$ denote the subset of elements $w \in W$ that have minimal length in the coset $W_{K} w$. Then ${ }^{K} W$ is a set of representatives of $W_{K} \backslash W$. The longest element in the set ${ }^{K} W$ is $w_{0, K} w_{0}$.

$\circ X_{+}^{*}(T)$ denotes the set of dominant characters - that is, characters $\lambda \in X^{*}(T)$ - such that $\left\langle\lambda, \alpha^{\vee}\right\rangle \geq 0$ for all $\alpha \in \Delta$.

$\circ$ For a subset $I \subset \Delta$, let $X_{+, I}^{*}(T)$ denote the set of characters $\lambda \in X^{*}(T)$ such that $\left\langle\lambda, \alpha^{\vee}\right\rangle \geq 0$ for all $\alpha \in I$. We call them $I$-dominant characters.

Definition 2.1.1. Let $P \subset G_{k}$ be a parabolic subgroup containing $B$ and let $L \subset P$ be the unique Levi subgroup of $P$ containing $T$. Then we define a subset $I_{P} \subset \Delta$ as the unique subset such that $W(L, T)=W_{I_{P}}$. For an arbitrary parabolic subgroup $P \subset G_{k}$ containing $T$, we put $I_{P}=I_{P^{\prime}} \subset \Delta$, where $P^{\prime}$ is the unique conjugate of $P$ containing $B$.

○ For a parabolic $P \subset G_{k}$, we put $\Delta^{P}=\Delta \backslash I_{P}$.

\subsection{The stack of G-zips}

In this section, we recall some facts about the stack of $G$-zips of Pink-Wedhorn-Ziegler.

\subsubsection{Zip datum}

Let $G$ be a connected reductive group over $\mathbb{F}_{q}$. In this article, a zip datum is a tuple $\mathcal{Z}=(G, P, L, Q, M, \varphi)$ consisting of the following objects:

(i) $P \subset G_{k}$ and $Q \subset G_{k}$ are parabolic subgroups of $G_{k}$.

(ii) $L \subset P$ and $M \subset Q$ are Levi subgroups such that $L^{(q)}=M$. In particular, the $q$-power Frobenius isogeny induces an isogeny $\varphi: L \rightarrow M$.

If $H$ is an algebraic group, denote by $R_{\mathrm{u}}(H)$ the unipotent radical of $H$. For $x \in P$, we can write uniquely $x=\bar{x} u$ with $\bar{x} \in L$ and $u \in R_{\mathrm{u}}(P)$. This defines a projection map $\theta_{L}^{P}: P \rightarrow L ; x \mapsto \bar{x}$. Similarly, we have a projection $\theta_{M}^{Q}: Q \rightarrow M$. The zip group is the subgroup of $P \times Q$ defined by

$$
E:=\left\{(x, y) \in P \times Q \mid \varphi\left(\theta_{L}^{P}(x)\right)=\theta_{M}^{Q}(y)\right\} .
$$

In other words, $E$ is the subgroup of $P \times Q$ generated by $R_{\mathrm{u}}(P) \times R_{\mathrm{u}}(Q)$ and elements of the form $(a, \varphi(a))$ with $a \in L$. Let $G \times G$ act on $G_{k}$ by $(a, b) \cdot g:=a g b^{-1}$, and let $E$ act on $G$ by restricting this action to $E$. The stack of $G$-zips of type $z$ can be defined as the quotient stack

$$
G-\operatorname{Zip}^{z}=\left[E \backslash G_{k}\right]
$$

Although the above definition of $G$-Zip ${ }^{2}$ may be the most concise one, there is a more useful, equivalent definition in terms of torsors: By [PWZ15, §3C and 3D], the stack $G-Z$ ip $^{2}$ is the stack over $k$ such that for all $k$-scheme $S$, the groupoid $G$ - $\operatorname{Zip}(S)$ is the category of tuples $\underline{\mathcal{J}}=\left(\mathcal{J}, \mathcal{J}_{P}, \mathcal{J}_{Q}, \iota\right)$, where $\mathcal{J}$ 
is a $G_{k}$-torsor over $S, \mathcal{J}_{P} \subset \mathcal{J}$ and $\mathcal{J}_{Q} \subset \mathcal{J}$ are a $P$-subtorsor and a $Q$-subtorsor of $\mathcal{J}$ respectively and $\iota:\left(\mathcal{J}_{P} / R_{\mathrm{u}}(P)\right)^{(p)} \rightarrow \mathcal{J}_{Q} / R_{\mathrm{u}}(Q)$ is an isomorphism of $M$-torsors.

\subsubsection{Cocharacter datum}

A convenient way to give a zip datum is using cocharacters. A cocharacter datum is a pair $(G, \mu)$ where $G$ is a reductive connected group over $\mathbb{F}_{q}$ and $\mu: \mathbb{G}_{\mathrm{m}, k} \rightarrow G_{k}$ is a cocharacter. There is a natural way to attach to $(G, \mu)$ a zip datum $z_{\mu}$, defined as follows. First, denote by $P_{+}(\mu)$ (respectively $\left.P_{-}(\mu)\right)$ the unique parabolic subgroup of $G_{k}$ such that $P_{+}(\mu)(k)$ (respectively $P_{-}(\mu)(k)$ ) consists of the elements $g \in G(k)$ satisfying that the map

$$
\mathbb{G}_{\mathrm{m}, k} \rightarrow G_{k} ; t \mapsto \mu(t) g \mu(t)^{-1} \quad\left(\text { respectively } t \mapsto \mu(t)^{-1} g \mu(t)\right)
$$

extends to a morphism of varieties $\mathbb{A}_{k}^{1} \rightarrow G_{k}$. This construction yields a pair of parabolics $\left(P_{+}(\mu), P_{-}(\mu)\right)$ in $G_{k}$ such that the intersection $P_{+}(\mu) \cap P_{-}(\mu)=L(\mu)$ is the centraliser of $\mu$. It is a common Levi subgroup of $P_{+}(\mu)$ and $P_{-}(\mu)$. Set $P=P_{-}(\mu), Q=\left(P_{+}(\mu)\right)^{(q)}, L=L(\mu)$ and $M=(L(\mu))^{(q)}$. Then the tuple $z_{\mu}:=(G, P, L, Q, M, \varphi)$ is a zip datum, which we call the zip datum attached to the cocharacter datum $(G, \mu)$. We write simply $G$-Zip ${ }^{\mu}$ for $G$-Zip ${ }^{z_{\mu}}$. For simplicity, we will always consider zip data arising in this way from a cocharacter datum.

\subsubsection{Frames}

In this article, given a zip datum $\mathcal{Z}=(G, P, L, Q, M, \varphi)$, a frame for $\mathcal{Z}$ is a triple $(B, T, z)$ where $(B, T)$ is a Borel pair of $G_{k}$ defined over $\mathbb{F}_{q}$ satisfying the following conditions:

(i) One has the inclusion $B \subset P$.

(ii) $z \in W$ is an element satisfying the conditions

$$
{ }^{z} B \subset Q \text { and } B \cap M={ }^{z} B \cap M .
$$

Remark 2.2.1. Let $(B, T)$ be a Borel pair defined over $\mathbb{F}_{q}$ such that $B \subset P$. Then we can find $z \in W$ such that $(B, T, z)$ is a frame. This follows from the proof of [PWZ11, Proposition 3.7].

A frame may not always exist. However, if $(G, \mu)$ is a cocharacter datum and $z_{\mu}$ is the associated zip datum (Subsection 2.2.2), then we can find a $G(k)$-conjugate $\mu^{\prime}=\operatorname{ad}(g) \circ \mu$ (with $g \in G(k)$ ) such that $z_{\mu^{\prime}}$ admits a frame. This follows easily from Remark 2.2.1 and the fact that $G$ is quasi-split over $\mathbb{F}_{q}$. Hence, it is harmless to assume that a frame exists, and we will only consider a zip datum that admits a frame.

Remark 2.2.2. If the cocharacter $\mu$ is defined over $\mathbb{F}_{q}$, then so are $P$ and $Q$. In particular, we have in this case $L=M$ and $P, Q$ are opposite parabolic subgroups with common Levi subgroup $L$.

For a zip datum $(G, P, L, Q, M, \varphi)$, we put $I=I_{P} \subset \Delta$. Note that $\Delta^{P}=\Delta \backslash I$.

Lemma 2.2.3 ([GK19b, Lemma 2.3.4]). Let $\mu: \mathbb{G}_{m, k} \rightarrow G_{k}$ be a cocharacter, and let $z_{\mu}$ be the attached zip datum. Assume that $(B, T)$ is a Borel pair defined over $\mathbb{F}_{q}$ such that $B \subset P$. We put $z=\sigma\left(w_{0, I}\right) w_{0}$. Then $(B, T, z)$ is a frame for $z_{\mu}$.

\subsubsection{Parametrisation of $E$-orbits}

Recall that the group $E$ from (2.2.1) acts on $G_{k}$. We review below the parametrisation of $E$-orbits following [PWZ11].

Assume that $z$ has a frame $(B, T, z)$. For $w \in W$, fix a representative $\dot{w} \in N_{G}(T)$, such that $\left(w_{1} w_{2}\right)^{\cdot}=\dot{w}_{1} \dot{w}_{2}$ whenever $\ell\left(w_{1} w_{2}\right)=\ell\left(w_{1}\right)+\ell\left(w_{2}\right)$ (this is possible by choosing a Chevalley system, [ABD+66, Exposé XXIII, §6]). For $w \in W$, define $G_{w}$ as the $E$-orbit of $\dot{w} \dot{z}^{-1}$. We note that $G_{w}$ is independent of the choices of $\dot{w}$ and a frame by [PWZ11, Proposition 5.8]. If no confusion occurs, we write $w$ instead of $\dot{w}$. Define a twisted order on ${ }^{I} W$ as follows. For $w, w^{\prime} \in{ }^{I} W$, write $w^{\prime} \leqslant w$ if there exists $w_{1} \in W_{L}$ such that $w^{\prime} \leq w_{1} w \sigma\left(w_{1}\right)^{-1}$. This defines a partial order on ${ }^{I} W$ [PWZ11, Corollary 6.3]. 
Theorem 2.2.4 ([PWZ11, Theorem 6.2, Theorem 7.5]). The map $w \mapsto G_{w}$ restricts to a bijection

$$
{ }^{I} W \rightarrow\left\{\text { E-orbits in } G_{k}\right\}
$$

For $w \in{ }^{I} W$, one has $\operatorname{dim}\left(G_{w}\right)=\ell(w)+\operatorname{dim}(P)$. Furthermore, for $w \in{ }^{I} W$, the Zariski closure of $G_{w}$ is

$$
\bar{G}_{w}=\bigsqcup_{w^{\prime} \in I} \bigsqcup_{W, w^{\prime} \leqslant w} G_{w^{\prime}}
$$

Each $E$-orbit is locally closed in $G_{k}$. Because $E$ is smooth over $k$, all $E$-orbits are also smooth over $k$. However, the Zariski closure $\bar{G}_{w}$ of $G_{w}$ may have highly complicated singularities; see [Kos 18 ] for a description of the normalisation of $\bar{G}_{w}$. The closure of an $E$-orbit is a union of $E$-orbits; hence we obtain a stratification of $G$.

In particular, there is a unique open $E$-orbit $U_{z} \subset G_{k}$ corresponding to the longest element $w_{0, I} w_{0} \in$ ${ }^{I} W$ via (2.2.2). For an $E$-orbit $G_{w}$ (with $w \in{ }^{I} W$ ), we write $X_{w}:=\left[E \backslash G_{w}\right]$ for the corresponding locally closed substack of $G$-Zip ${ }^{z}=\left[E \backslash G_{k}\right]$.

If $Z$ arises from a cocharacter datum (Subsection 2.2.2), we write $U_{\mu}$ for $U_{z_{\mu}}$. Using the terminology pertaining to the theory of Shimura varieties, we call $U_{\mu}$ the $\mu$-ordinary stratum of $G$-Zip ${ }^{\mu}$. The corresponding substack $\mathcal{U}_{\mu}:=\left[E \backslash U_{\mu}\right]$ is called the $\mu$-ordinary locus. It corresponds to the $\mu$-ordinary locus in the good reduction of Shimura varieties, studied, for example, in [Wor13], [Moo04]. For more details about Shimura varieties, we refer to Subsection 2.5.

\subsection{Reminders about representation theory}

If $H$ is an algebraic group over a field $K$, denote by $\operatorname{Rep}(H)$ the category of algebraic representations of $H$ on finite-dimensional $K$-vector spaces. We will denote such a representation by $(V, \rho)$ or sometimes simply $\rho$ or $V$.

Let $H$ be a split connected reductive $K$-group and choose a Borel pair $\left(B_{H}, T\right)$ defined over $K$. Irreducible representations of $H$ are in one-to-one correspondence with dominant characters $X_{+}^{*}(T)$. This bijection is given by the highest weight of a representation. For $\lambda \in X_{+}^{*}(T)$, let $\mathcal{L}_{\lambda}$ be the line bundle attached to $\lambda$ on the flag variety $H / B_{H}$ by the usual associated sheaf construction [Jan03, §5.8]. Define an $H$-representation $V_{H}(\lambda)$ by

$$
V_{H}(\lambda):=H^{0}\left(H / B_{H}, \mathcal{L}_{\lambda}\right)
$$

In other words, $V_{H}(\lambda)$ is the induced representation $\operatorname{Ind}_{B_{H}}^{H} \lambda$. Then $V_{H}(\lambda)$ is a representation of highest weight $\lambda$. We view elements of $V_{H}(\lambda)$ as functions $f: H \rightarrow \mathbb{A}^{1}$ satisfying the relation

$$
f(h b)=\lambda\left(b^{-1}\right) f(h), \quad \forall h \in H, \forall b \in B_{H} .
$$

For dominant characters $\lambda, \lambda^{\prime}$, there is a natural surjective map

$$
V_{H}(\lambda) \otimes V_{H}\left(\lambda^{\prime}\right) \rightarrow V_{H}\left(\lambda+\lambda^{\prime}\right)
$$

In the description given by (2.3.2), this map is simply given by mapping $f \otimes f^{\prime}$ (where $f \in V_{H}(\lambda)$, $\left.f^{\prime} \in V_{H}\left(\lambda^{\prime}\right)\right)$ to the function $f f^{\prime} \in V_{H}\left(\lambda+\lambda^{\prime}\right)$.

Denote by $W_{H}:=W(H, T)$ the Weyl group and $w_{0, H} \in W_{H}$ the longest element. Then $V_{H}(\lambda)$ has a unique $B_{H}$-stable line, which is a weight space for the weight $w_{0, H} \lambda$. 


\subsection{Vector bundles on the stack of G-zips}

\subsubsection{General theory}

For an algebraic stack $X$, write $\mathfrak{B B}(X)$ for the category of vector bundles on $X$. Let $X$ be a $k$-scheme and $H$ an affine $k$-group scheme acting on $X$. If $\rho: H \rightarrow \mathrm{GL}(V)$ is a finite-dimensional algebraic representation of $H$, it gives rise to a vector bundle $\mathcal{V}_{H, X}(\rho)$ on the stack $[H \backslash X]$. This vector bundle can be defined geometrically as $\left[H \backslash\left(X \times_{k} V\right)\right]$, where $H$ acts diagonally on $X \times_{k} V$. We obtain a functor

$$
\mathcal{V}_{H, X}: \operatorname{Rep}(H) \rightarrow \mathfrak{B B}([H \backslash X]) .
$$

In particular, similar to the usual associated sheaf constrution [Jan03, Chapter I, §5.8, Equation (1)], the space of global sections $H^{0}\left([H \backslash X], \mathcal{V}_{H, X}(\rho)\right)$ is identified with

$$
H^{0}\left([H \backslash X], \mathcal{V}_{H, X}(\rho)\right)=\{f: X \rightarrow V \mid f(h \cdot x)=\rho(h) f(x), \quad \forall h \in H, \forall x \in X\} .
$$

\subsubsection{Automorphic vector bundles on $G$-Zip ${ }^{2}$}

Fix a zip datum

$$
z=(G, P, L, Q, M, \varphi)
$$

and a frame $(B, T, z)$ as usual. By the previous paragraph, we obtain a functor $\mathcal{V}_{E, G}: \operatorname{Rep}(E) \rightarrow$ $\mathfrak{B} \mathfrak{B}\left(G\right.$-Zip $\left.{ }^{z}\right)$, which we simply denote by $\mathcal{V}$. For $(V, \rho) \in \operatorname{Rep}(E)$, the space of global sections of $\mathcal{V}(\rho)$ is

$$
H^{0}\left(G-\mathrm{Zip}^{2}, \mathcal{V}(\rho)\right)=\left\{f: G_{k} \rightarrow V \mid f(\epsilon \cdot g)=\rho(\epsilon) f(g), \quad \forall \epsilon \in E, \forall g \in G_{k}\right\} .
$$

One has the following easy lemma, which follows from the fact that $G_{k}$ admits an open dense $E$-orbit (see discussion below Theorem 2.2.4).

Lemma 2.4.1 ([Kos19, Lemma 1.2.1]). Let $(V, \rho)$ be an E-representation. Then we have $\operatorname{dim} H^{0}\left(G-\operatorname{Zip}^{2}, \mathcal{V}(\rho)\right) \leq \operatorname{dim}(V)$.

The first projection $p_{1}: E \rightarrow P$ induces a functor $p_{1}^{*}: \operatorname{Rep}(P) \rightarrow \operatorname{Rep}(E)$. If $(V, \rho) \in$ $\operatorname{Rep}(P)$, we write again $\mathcal{V}(\rho)$ for $\mathcal{V}\left(p_{1}^{*}(\rho)\right)$. Let $\mathfrak{B B}_{P}\left(G\right.$-Zip $\left.{ }^{z}\right)$ be the essentail image of $\mathcal{V}: \operatorname{Rep}(P) \rightarrow \mathfrak{B B}\left(G-\right.$ Zip $\left.^{2}\right)$. We call $\mathfrak{B B}_{P}\left(G-\right.$ Zip $\left.^{z}\right)$ the category of automorphic vector bundles (cf. [Mil90, Chapter III, Remark 2.3]). The goal of this article is to study the vector bundles $\mathcal{V}(\rho)$ on $G$-Zip ${ }^{\mathcal{Z}}$ and determine their properties for $\rho \in \operatorname{Rep}(P)$. In particular, we seek to understand the properties of $\mathcal{V}(\rho)$ in terms of the representation $(V, \rho)$ defining it.

\subsection{3. $L$-representations}

Let $\theta_{L}^{P}: P \rightarrow L$ denote again the natural projection modulo the unipotent radical $R_{\mathrm{u}}(P)$, as in Subsection 2.2.1. It induces by composition a functor

$$
\left(\theta_{L}^{P}\right)^{*}: \operatorname{Rep}(L) \rightarrow \operatorname{Rep}(P) .
$$

It is easy to see that $\left(\theta_{L}^{P}\right)^{*}$ is a fully faithful functor, and its image is the full subcategory of $\operatorname{Rep}(P)$ of $P$-representations that are trivial on $R_{\mathrm{u}}(P)$. Hence, we view $\operatorname{Rep}(L)$ as a full subcategory of $\operatorname{Rep}(P)$. If $(V, \rho) \in \operatorname{Rep}(L)$, we write again $\mathcal{V}(\rho):=\mathcal{V}\left(\left(\theta_{L}^{P}\right)^{*}(\rho)\right)$. For $\lambda \in X_{+, I}^{*}(T)$, write $B_{L}:=B \cap L$ and define an $L$-representation as

$$
V_{I}(\lambda)=\operatorname{Ind}_{B_{L}}^{L}(\lambda)
$$

This is the representation defined in (2.3.1) for $H=L$ and $B_{H}=B_{L}$. Denote by $\mathcal{V}_{I}(\lambda)$ the vector bundle on $G$-Zip ${ }^{z}$ attached to $V_{I}(\lambda)$. We call $\mathcal{V}_{I}(\lambda)$ the automorphic vector bundle associated to the weight $\lambda$ on $G$-Zip ${ }^{2}$. This terminology stems from Shimura varieties (see Subsection 2.5 for further details). Note that if $\lambda \in X^{*}(T)$ is not $L$-dominant, then $V_{I}(\lambda)=0$ and hence $\mathcal{V}_{I}(\lambda)=0$. In [Kos19], the 
second author studied the vector bundles $\mathcal{V}_{I}(\lambda)$ on $G$-Zip ${ }^{2}$. In particular, he investigated the question of determining the set $C_{\text {zip }}$ of characters $\lambda \in X_{+, I}^{*}(T)$ such that the space $H^{0}\left(G\right.$-Zip $\left.{ }^{z}, \mathcal{V}_{I}(\lambda)\right)$ is nonzero. In a work in progress [GIK21] with Goldring, we completely determine $C_{\text {zip }}$ under the condition that $P$ is defined over $\mathbb{F}_{q}$ and the Frobenius $\sigma$ acts on $I$ by $-w_{0, I}$.

\subsection{Shimura varieties}

In this subsection, we explain the link between the stack of $G$-zips and Shimura varieties. Let $(\mathbf{G}, \mathbf{X})$ be a Shimura datum [De179, §2.1.1]. In particular, $\mathbf{G}$ is a connected reductive group over $\mathbb{Q}$. Furthermore, $\mathbf{X}$ provides a well-defined $\mathbf{G}(\overline{\mathbb{Q}})$-conjugacy class $\{\mu\}$ of cocharacters of $\mathbf{G}_{\overline{\mathbb{Q}}}$. Write $\mathbf{E}=E(\mathbf{G}, \mathbf{X})$ for the reflex field of $(\mathbf{G}, \mathbf{X})$ (i.e., the field of definition of $\{\mu\})$ and $\mathcal{O}_{\mathbf{E}}$ for its ring of integers. Given an open compact subgroup $K \subset \mathbf{G}\left(\mathbf{A}_{f}\right)$, write $\operatorname{Sh}(\mathbf{G}, \mathbf{X})_{K}$ for the canonical model at level $K$ over $\mathbf{E}$ (cf. [Del79, §2.2]). For $K$ small enough in $\mathbf{G}\left(\mathbb{A}_{f}\right), \operatorname{Sh}(\mathbf{G}, \mathbf{X})_{K}$ is a smooth, quasi-projective scheme over E. For a small enough $K$, every inclusion $K^{\prime} \subset K$ induces a finite étale projection $\pi_{K^{\prime} / K}: \operatorname{Sh}(\mathbf{G}, \mathbf{X})_{K^{\prime}} \rightarrow \operatorname{Sh}(\mathbf{G}, \mathbf{X})_{K}$.

Let $g \geq 1$ and let $(V, \psi)$ be a $2 g$-dimensional, nondegenerate symplectic space over $\mathbb{Q}$. Write $\operatorname{GSp}(2 g)=\operatorname{GSp}(V, \psi)$ for the group of symplectic similitudes of $(V, \psi)$. Write $\mathbf{X}_{g}$ for the double Siegel half-space [Del79, §1.3.1]. The pair $\left(\operatorname{GSp}(2 g), \mathbf{X}_{g}\right)$ is called the Siegel-Shimura datum and has reflex field $\mathbb{Q}$. Recall that $(\mathbf{G}, \mathbf{X})$ is of Hodge type if there exists an embedding of Shimura data $\iota:(\mathbf{G}, \mathbf{X}) \hookrightarrow\left(\mathrm{GSp}(2 g), \mathbf{X}_{g}\right)$ for some $g \geq 1$. Henceforth, assume that $(\mathbf{G}, \mathbf{X})$ is of Hodge type.

Fix a prime number $p$ and assume that the level $K$ is of the form $K=K_{p} K^{p}$ where $K_{p} \subset \mathbf{G}\left(\mathbb{Q}_{p}\right)$ is a hyperspecial subgroup and $K^{p} \subset \mathbf{G}\left(\mathbb{A}_{f}^{p}\right)$ is an open compact subgroup. Recall that a hyperspecial subgroup of $\mathbf{G}\left(\mathbb{Q}_{p}\right)$ exists if and only if $\mathbf{G}_{\mathbb{Q}_{p}}$ is unramified and is of the form $K_{p}=\mathscr{G}\left(\mathbb{Z}_{p}\right)$ where $\mathscr{G}$ is a reductive group over $\mathbb{Z}_{p}$ such that $\mathscr{G} \otimes_{\mathbb{Z}_{p}} \mathbb{Q}_{p} \simeq \mathbf{G}_{\mathbb{Q}_{p}}$ and $\mathscr{G} \otimes_{\mathbb{Z}_{p}} \mathbb{F}_{p}$ is connected.

We assume that $p>2$. For any place $v$ above $p$ in $\mathbf{E}$, Kisin [Kis10] and Vasiu [Vas99] constructed a family of smooth $\mathcal{O}_{\mathbf{E}_{v}}$-schemes $\mathcal{S}=\left(\mathcal{S}_{K}\right)_{K^{p}}$, where $K=K_{p} K^{p}$ and $K^{p}$ is a small enough compact open subgroup of $\mathbf{G}\left(\mathbb{A}_{f}^{p}\right)$. For $K^{\prime p} \subset K^{p}$, one has again a finite étale projection $\pi_{K^{\prime} / K}: \mathcal{S}_{K_{p} K^{\prime}} \rightarrow \mathcal{S}_{K_{p} K^{p}}$, where $K=K_{p} K^{p}$ and $K^{\prime}=K_{p} K^{\prime p}$ and the tower $\mathcal{S}=\left(\mathcal{S}_{K}\right)_{K^{p}}$ is an $\mathcal{O}_{\mathbf{E}_{v}}$-model of the tower $\left(\operatorname{Sh}(\mathbf{G}, \mathbf{X})_{K}\right)_{K}$. We write $S_{K}$ for the geometric special fibre of $\mathcal{S}_{K}$.

We take a representative $\mu \in\{\mu\}$ defined over $\mathbf{E}_{v}$ by [Kot84, (1.1.3) Lemma (a)]. We can also assume that $\mu$ extends to $\mu: \mathbb{G}_{\mathrm{m}, \mathcal{O}_{\mathbf{E}_{v}}} \rightarrow \mathscr{G}_{\mathcal{O}_{\mathbf{E}_{v}}}$ [Kim18, Corollary 3.3.11]. Denote by $\mathbf{L} \subset \mathbf{G}_{\mathbf{E}_{v}}$ the centraliser of the cocharacter $\mu$. We take a parabolic sugroups $\mathbf{P}$ of $\mathbf{G}_{\mathbf{E}_{v}}$, which has $\mathbf{L}$ as a Levi subgroup. Since $\mathbf{G}_{\mathbb{Q}_{p}}$ is unramified, it is quasi-split, hence we can choose a Borel subgroup $\mathbf{B} \subset \mathbf{G}_{\mathbb{Q}_{p}}$ and a maximal torus $\mathbf{T} \subset \mathbf{B}$. There is $g \in \mathbf{G}\left(\mathbf{E}_{v}\right)$ such that $\mathbf{B}_{\mathbf{E}_{v}} \subset g \mathbf{P} g^{-1}$. Write $g=b g_{0}$ with $b \in B\left(\mathbf{E}_{v}\right)$ and $g_{0} \in \mathscr{G}\left(\mathcal{O}_{\mathbf{E}_{v}}\right)$ by the Iwasawa decomposition. Then replacing $\mu$ by its conjugate by $g_{0}$, we may assume that $\mathbf{B}_{\mathbf{E}_{v}} \subset \mathbf{P}$.

By properness of the scheme of parabolic subgroups of $\mathscr{G}$ [ABD+66, Exposé XXVI, Corollaire 3.5], the subgroups $\mathbf{B}$ and $\mathbf{P}$ extend uniquely to subgroups $\mathscr{B} \subset \mathscr{G}$ over $\mathbb{Z}_{p}$ and $\mathscr{P} \subset \mathscr{G}_{\mathcal{O}_{\mathbf{E}_{v}}}$ over $\mathcal{O}_{\mathbf{E}_{v}}$ respectively. Let $\mathscr{L} \subset \mathscr{P}$ be the centraliser of $\mu: \mathbb{G}_{\mathrm{m}, \mathcal{O}_{\mathbf{E}_{v}}} \rightarrow \mathscr{G}_{\mathcal{O}_{\mathbf{E}_{v}}}$. We take a Borel subgroup $\mathbf{B}^{\text {op }}$ of $\mathbf{G}_{\mathbb{Q}_{p}}$ such that $\mathbf{T}=\mathbf{B} \cap \mathbf{B}^{\text {op }}$. The subgroup $\mathbf{B}^{\text {op }}$ extends uniquely to a subgroup $\mathscr{B}^{\text {op }} \subset \mathscr{G}$ over $\mathbb{Z}_{p}$. We put $\mathscr{T}=\mathscr{B} \cap \mathscr{B}$ op. Set $G=\mathscr{G} \otimes_{\mathbb{Z}_{p}} \mathbb{F}_{p}$ and denote by $B, T, P, L$ the geometric special fibre of $\mathscr{B}, \mathscr{T}, \mathscr{P}, \mathscr{L}$ respectively. By slight abuse of notation, we denote again by $\mu$ its $\bmod p$ reduction $\mu: \mathbb{G}_{\mathrm{m}, k} \rightarrow G_{k}$. Then $(G, \mu)$ is a cocharacter datum, and it yields a zip datum $(G, P, L, Q, M, \varphi)$ as in Subsection 2.2.2 (because $G$ is defined over $\mathbb{F}_{p}$, in the context of Shimura varieties, we always take $q=p$; hence, $\varphi$ is the $p$ th power Frobenius).

By a result of Zhang [Zha18, 4.1], there exists a natural smooth morphism

$$
\zeta: S_{K} \rightarrow G-\mathrm{Zip}^{\mu} .
$$

This map is also surjective by [SYZ19, Corollary 3.5.3(1)]. The map $\zeta$ amounts to the existence of a universal $G$-zip $\underline{\mathcal{J}}=\left(\mathcal{J} \mathcal{J}_{P}, \mathcal{J}_{Q}, \iota\right)$ over $S_{K}$, using the description of $G$-Zip ${ }^{\mu}$ provided at the end of Subsection 2.2.1. In the construction of Zhang, the $G_{k}$-torsor J and the $P$-torsor $\mathcal{J}_{P}$ over $S_{K}$ are actually the reduction of a $\mathscr{G}$-torsor and a $\mathscr{P}$-torsor over $\mathcal{S}_{K}$, which we denote by $\mathscr{I}$ and $\mathscr{I}_{\mathscr{P}}$ respectively. 
Example 2.5.1. We explain the example of the Siegel-type Shimura variety. In this case, one has $\mathbf{G}=\operatorname{GSp}(V, \psi)$ for a symplectic space $(V, \psi)$ of dimension $2 g(g \geq 1)$ over $\mathbb{Q}$. The $\mathbb{Z}_{p}$-model $\mathscr{G}=\operatorname{GSp}(\Lambda, \psi)$ is given by a self-dual $\mathbb{Z}_{p}$-lattice $\Lambda \subset V_{\mathbb{Q}_{p}}$; that is, a lattice satisfying $\Lambda^{\vee}=\Lambda$, where $\Lambda^{\vee}:=\left\{x \in V_{\mathbb{Q}_{p}} \mid \forall y \in \Lambda, \psi(x, y) \in \mathbb{Z}_{p}\right\}$. The cocharacter $\mu: \mathbb{G}_{\mathrm{m}, \mathbb{Z}_{p}} \rightarrow \mathbf{G}_{\mathbb{Z}_{p}}$ induces a decomposition $\Lambda=\Lambda_{0} \oplus \Lambda_{1}$, where $\Lambda_{0}, \Lambda_{1}$ are free $\mathbb{Z}_{p}$-modules of rank $g$. Here $z \in \mathbb{G}_{\mathrm{m}}$ acts via $\mu$ on $\Lambda_{i}$ by the character $z \mapsto z^{i}$ for $i \in\{0,1\}$. Define two filtrations

$$
\begin{aligned}
& \operatorname{Fil}_{0}(\Lambda): 0 \subset \Lambda_{0} \subset \Lambda \text { and } \\
& \operatorname{Fil}_{1}(\Lambda): 0 \subset \Lambda_{1} \subset \Lambda .
\end{aligned}
$$

Then $\mathscr{P}$ can be defined as the parabolic subgroup of $\mathscr{G}$ stabilising $\operatorname{Fil}_{0}(\Lambda)$. The scheme $\mathcal{S}_{K}$ (with $K=K_{p} K^{p}$ and $K_{p}=\mathscr{G}\left(\mathbb{Z}_{p}\right)$ as above $)$ is a moduli space classifying triples $\left(A, \xi, \eta K^{p}\right)$ where $A$ is an abelian variety of rank $g$ endowed with a principal polarisation $\xi$ and a $K^{p}$-level structure $\eta K^{p}$. Here $\eta$ is a symplectic isomorphism $H^{1}\left(A, \mathbb{A}^{p}\right) \simeq V \otimes \mathbb{A}^{p}$ and $\eta K^{p}$ is its $K^{p}$-coset in the set of such isomorphisms.

Let $\mathscr{A} \rightarrow \delta_{K}$ denote the universal abelian scheme. Then

$$
\mathscr{H}:=H_{\mathrm{dR}}^{1}\left(\mathscr{A} / \mathcal{S}_{K}\right)
$$

is a rank $2 g$ vector bundle on $\mathcal{S}_{K}$, and the principal polarisation $\xi$ induces on $\mathscr{H}$ a perfect, symplectic pairing, which we denote by $\psi_{\xi}$. The vector bundle $\mathscr{H}$ also carries a natural Hodge filtration (which we denote by $\mathrm{Fil}_{\mathrm{Hdg}}$ ):

$$
0 \subset \Omega_{\mathscr{A} / \mathcal{S}_{K}} \subset \mathscr{H}
$$

where $\Omega_{\mathscr{A} / \mathcal{S}_{K}}$ is the push-forward of the sheaf of relative Kähler differentials $\Omega_{\mathscr{A} / \mathcal{S}_{K}}^{1}$ by the structural morphism $f: \mathscr{A} \rightarrow \mathcal{S}_{K}$. It is a rank $g$-subbundle of $\mathscr{H}$. We obtain a $\mathscr{G}$-torsor $\mathscr{I}$ and a $\mathscr{P}$-torsor $\mathscr{I}_{\mathscr{P}}$ over $\mathcal{S}_{K}$ as follows: For an $\mathcal{S}_{K}$-scheme $S$, we define $\mathscr{I}(S)$ by

$$
\underline{\operatorname{Isom}}_{\mathcal{O}_{S}}\left(\left(\Lambda \otimes \mathcal{O}_{S}, \psi\right),\left(\mathscr{H} \otimes_{\mathcal{O}_{\delta_{K}}} \mathcal{O}_{S}, \psi_{\xi}\right)\right)
$$

and $\mathscr{I}_{\mathscr{P}}(S)$ by

$$
\underline{\operatorname{Isom}}_{\mathcal{O}_{S}}\left(\left(\Lambda \otimes \mathcal{O}_{S}, \psi, \operatorname{Fil}_{0}(\Lambda) \otimes \mathcal{O}_{S}\right),\left(\mathscr{H} \otimes_{\mathcal{O}_{\delta_{K}}} \mathcal{O}_{S}, \psi_{\xi}, \mathrm{Fil}_{\mathrm{Hdg}} \otimes_{\mathcal{O}_{\delta_{K}}} \mathcal{O}_{S}\right)\right) .
$$

This defines two fppf sheaves on $\mathcal{S}_{K}$. Furthermore, $\mathscr{G}$ acts naturally on $\mathscr{I}$ via its action on $\Lambda$. Furthermore, because the parabolic group $\mathscr{P} \subset \mathscr{G}$ stabilises Fil $_{0}(\Lambda)$, the group $\mathscr{P}$ acts naturally on $\mathscr{I}_{\mathscr{P}}$. This defines respectively a $\mathscr{G}$-torsor and a $\mathscr{P}$-torsor on $\mathcal{S}_{K}$.

Over $S_{K}=\mathcal{S}_{K} \otimes \mathbb{F}_{p}$, the $G$-zip $\underline{\mathcal{J}}=\left(\mathcal{J}_{,} \mathcal{J}_{P}, \mathcal{J}_{Q}, \iota\right)$ is defined as follows. First define $\mathcal{J}$ and $\mathcal{J}_{P}$ to be the base change to $S_{K}$ of $\mathscr{I}$ and $\mathscr{I}_{\mathscr{P}}$. To define the $Q$-torsor $\mathcal{J}_{Q}$, recall that $H:=H_{\mathrm{dR}}^{1}\left(A / S_{K}\right)$ admits a conjugate filtration Fil conj $\subset H$ : Let $f: A \rightarrow S_{K}$ denote the universal abelian scheme (with $A:=$ $\left.\mathscr{A} \otimes_{\mathcal{S}_{K}} S_{K}\right)$, then there is a conjugate spectral sequence $E_{2}^{a b}=R^{a} f_{*}\left(\mathcal{H}^{b}\left(\Omega_{A / S_{K}}^{\bullet}\right)\right) \Rightarrow H_{\mathrm{dR}}^{a+b}\left(A / S_{K}\right)$. For abelian varieties, this spectral sequence degenerates and gives the filtration Fil conj on $H_{\mathrm{dR}}^{1}\left(A / S_{K}\right)$. Note that the conjugate filtration only exists on the special fibre of $\delta_{K}$, contrary to the Hodge filtration. For an $S_{K}$-scheme $S$, we put

$$
\mathcal{J}_{Q}(S)=\underline{\operatorname{Isom}}_{\mathcal{O}_{S}}\left(\left(\Lambda \otimes \mathcal{O}_{S}, \psi, \operatorname{Fil}_{1}(\Lambda) \otimes \mathcal{O}_{S}\right),\left(H \otimes_{\mathcal{O}_{S_{K}}} \mathcal{O}_{S}, \psi \xi, \operatorname{Fil}_{\text {conj }} \otimes_{\mathcal{O}_{S_{K}}} \mathcal{O}_{S}\right)\right) .
$$

Because $Q$ stabilises the filtration $\operatorname{Fil}_{1}(\Lambda) \otimes \mathbb{F}_{p}$, it acts naturally on $\mathcal{J}_{Q}$, and again we obtain a $Q$-torsor on $S_{K}$. Finally, the isomorphism $\iota:\left(\mathcal{J}_{P} / R_{\mathrm{u}}(P)\right)^{(p)} \rightarrow \mathcal{J}_{Q} / R_{\mathrm{u}}(Q)$ is naturally induced by the Frobenius and Verschiebung homomorphisms (or, more generally, the Cartier isomorphism; see [MW04, 7.3]). 
For each $\mathbf{L}$-dominant character $\lambda \in X^{*}(\mathbf{T})$, we have the unique irreducible representation $\mathbf{V}_{I}(\lambda)$ of $\mathbf{P}$ over $\overline{\mathbb{Q}}_{p}$ of highest weight $\lambda$. Because we are in characteristic zero, $\mathbf{V}_{I}(\lambda)$ coincides with $H^{0}\left(\mathbf{P} / \mathbf{B}, \mathcal{L}_{\lambda}\right)$, as defined in (2.3.1) in Subsection 2.3. It admits a natural model over $\overline{\mathbb{Z}}_{p}$, namely,

$$
\mathbf{V}_{I}(\lambda)_{\overline{\mathbb{Z}}_{p}}:=H^{0}\left(\mathscr{P} / \mathscr{B}, \mathcal{L}_{\lambda}\right),
$$

where $\mathcal{L}_{\lambda}$ is the line bundle attached to $\lambda$ viewed as a character of $\mathscr{T}$. Its reduction modulo $p$ is the $P$-representation $V_{I}(\lambda)=H^{0}\left(P / B, \mathcal{L}_{\lambda}\right)$ over $k=\overline{\mathbb{F}}_{p}$. Because $\delta_{K}$ is endowed naturally with a $\mathscr{P}$-torsor $\mathscr{I}_{\mathscr{P}}$, we obtain a vector bundle $\mathscr{V}_{I}(\lambda)$ on $\mathcal{S}_{K}$ by applying the $\mathscr{P}$-representation $\mathbf{V}_{I}(\lambda)_{\overline{\mathbb{Z}}_{p}}$ to $\mathscr{I}_{\mathscr{P}}$. The vector bundle $\mathscr{V}_{I}(\lambda)$ for $\lambda \in X^{*}(\mathbf{T})_{+, I}$ is called the automorphic vector bundle associated to the weight $\lambda$. For an $\mathcal{O}_{\mathbf{E}_{v}}$-algebra $R$, the space $H^{0}\left(\mathcal{S}_{K} \otimes_{\mathcal{O}_{\mathbf{E}_{v}}} R, \mathscr{V}_{I}(\lambda)\right)$ may be called the space of automorphic forms of level $K$ and weight $\lambda$ with coefficients in $R$. More generally, by the same formalism, we have a commutative diagram of functors

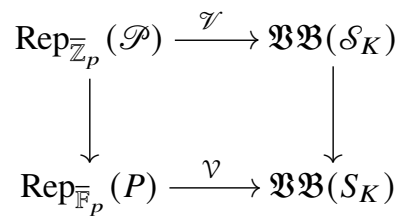

where the vertical arrows are reduction modulo $p$ and the horizontal arrows are obtained by applying the $\mathscr{P}_{\text {-torsor }} \mathscr{I}_{\mathscr{P}}$ and the $P$-torsor $\mathcal{J}_{P}$ respectively. The vector bundles obtained in this way on $\delta_{K}$ and $S_{K}$ are called automorphic vector bundles following [Mil90, Chapter III, Remark 2.3].

Furthermore, the map $\zeta: S_{K} \rightarrow G$-Zip ${ }^{\mu}$ induces a factorization of the lower horizontal arrow of the above diagram as

$$
\operatorname{Rep}_{\overline{\mathbb{F}}_{p}}(P) \stackrel{\mathcal{V}}{\longrightarrow} \mathfrak{B} \mathfrak{B}\left(G-\text { Zip }^{\mu}\right) \stackrel{\zeta^{*}}{\longrightarrow} \mathfrak{B B}\left(S_{K}\right) .
$$

Note also that for any $P$-representation $(V, \rho)$, the map $\zeta: S_{K} \rightarrow G$-Zip ${ }^{\mu}$ induces by pullback a natural injective morphism

$$
H^{0}\left(G-\operatorname{Zip}^{\mu}, \mathcal{V}(\rho)\right) \rightarrow H^{0}\left(S_{K}, \mathcal{V}(\rho)\right)
$$

In Section 3, we determine the space $H^{0}\left(G-\mathrm{Zip}^{\mu}, \mathcal{V}(\rho)\right)$ in all generality (i.e., even for cocharacter data $(G, \mu)$ that are not attached to Shimura varieties). For general pairs $(G, \mu)$ with $\mu$ minuscule (but not necessarily attached to Shimura varieties), one has the following remark.

Remark 2.5.2. Let $F$ be a local field with ring of integers $\mathcal{O}$ and residue field $\mathbb{F}_{q}$. Let $G$ be an unramified reductive group over $\mathcal{O}$. Let $(B, T)$ be a Borel pair of $G$, and let $\mu$ be a dominant cocharacter of $G$. Then Xiao-Zhu define the moduli of local shtukas Sht ${ }_{\mu}^{\text {loc }}$ classifying modifications bounded by $\mu$ of a $G$-torsor and its Frobenius twist (see [XZ17, Definition 5.2.1]). Similarly, there is a moduli $\operatorname{Sht}_{\mu} \operatorname{loc}(m, n)$ of restricted local shtuka [XZ17, §5.3], with a natural projection $\operatorname{Sht}_{\mu}^{\text {loc }} \rightarrow \operatorname{Sht}_{\mu}^{\text {loc }(m, n)}$. In the case when $\mu$ is minuscule, Xiao-Zhu show in [XZ17, Lemma 5.3.6] that there exists a natural perfectly smooth morphism Sht ${ }_{\mu}^{\text {loc }(2,1)} \rightarrow G$-Zip ${ }^{\mu, p f}$, where pf denotes the perfection and the special fibre of $G$ is again denoted by $G$ (see Subsection 3.5 for further details).

\section{The space of global sections $H^{0}\left(G-\mathrm{Zip}^{\mu}, \mathcal{V}(\rho)\right)$}

\subsection{Adapted morphisms}

To determine the space $H^{0}\left(G\right.$-Zip $\left.{ }^{\mu}, \mathcal{V}(\rho)\right)$ (for $(V, \rho)$ a $P$-representation), we use a similar method as in $[\operatorname{Kos} 19, \S 3.2]$, where we studied representations of the type $V_{I}(\lambda)$. We review some of the notions introduced in [Kos19, §3.2]. 
Let $X$ be an irreducible normal $k$-variety and let $U \subset X$ be an open subset such that $S=X \backslash U$ is irreducible of codimension 1. For $f \in H^{0}\left(U, \mathcal{O}_{X}\right)$, denote by $Z_{U}(f) \subset U$ the vanishing locus of $f$ in $U$ and let $\overline{Z_{U}(f)}$ be its Zariski closure in $X$. We endow all locally closed subsets of schemes with the reduced structure. Let $Y$ be an irreducible $k$-variety and $\psi: Y \rightarrow X$ be a $k$-morphism.

Definition 3.1.1. We say that $\psi$ is adapted to $f$ (with respect to $U$ ) if

(i) $\psi(Y) \cap U \neq \emptyset$ and

(ii) $\psi(Y) \cap S$ is not contained in $\overline{Z_{U}(f)}$.

Lemma 3.1.2. If $\psi(Y)$ intersects $U$ and $\psi(Y) \cap S$ is dense in $S$, then $\psi$ is adapted to any nonzero section $f \in H^{0}\left(U, \mathcal{O}_{X}\right)$.

Proof. We need to show that condition (ii) is satisfied. We may assume that $Z_{U}(f) \neq \emptyset$. Then, the closed subset $\overline{Z_{U}(f)}$ has codimension 1 in $X$ and intersects $U$; hence, $\overline{Z_{U}(f)} \cap S$ has codimension $\geq 1$ in $S$, so it cannot contain $\psi(Y) \cap S$.

Lemma 3.1.3 ([Kos19, Lemma 3.2.2]). Let $\psi: Y \rightarrow X$ be a morphism adapted to $f \in H^{0}\left(U, \mathcal{O}_{X}\right)$. Then $f$ extends to $X$ if and only if $\psi^{*}(f) \in H^{0}\left(\psi^{-1}(U), \mathcal{O}_{Y}\right)$ extends to $Y$. In this case, $f$ vanishes along $S$ if and only if $\psi^{*}(f)$ vanishes along $\psi^{-1}(S)$.

We apply the above notions to the following situation. From now on, let $(G, \mu)$ be a cocharacter datum, with attached zip datum $z=(G, P, L, Q, M, \varphi)$ as in Subsection 2.2.2. Assume that $(B, T)$ is a Borel pair defined over $\mathbb{F}_{q}$ such that $B \subset P$. We take a frame $(B, T, z)$ as in Lemma 2.2.3. Consider the variety $G_{k}$ and the open subset $U_{\mu} \subset G_{k}$ (the $\mu$-ordinary stratum, defined after Theorem 2.2.4). The complement of $U_{\mu}$ in $G_{k}$ is not irreducible in general, so in order to apply the previous results, we slightly modify the problem. Recall the parametrisation of $E$-orbits in $G_{k}$ (2.2.2). Using Theorem 2.2.4, we have

$$
G_{k} \backslash U_{\mu}=\bigcup_{\alpha \in \Delta^{P}} Z_{\alpha}, \quad Z_{\alpha}=\overline{E \cdot s_{\alpha}},
$$

where $E \cdot s_{\alpha}$ denotes the $E$-orbit of $s_{\alpha}$ and the bar denotes the Zariski closure. Indeed, by (2.2.2), the $E$-orbits of codimension 1 in $G_{k}$ are the $E$-orbits of $w z^{-1}$ where $w \in{ }^{I} W$ is an element of length $\ell\left(w_{0, I} w_{0}\right)-1$. These elements are of the form $w_{0, I} s_{\alpha} w_{0}$ for $\alpha \in \Delta^{P}$. Because $z=\sigma\left(w_{0, I}\right) w_{0}$, the element $w z^{-1}$ has the form $w_{0, I} s_{\alpha} \sigma\left(w_{0, I}\right)$. Because $\left(w_{0, I}, \sigma\left(w_{0, I}\right)\right) \in E$, this element generates the same $E$-orbit as $s_{\alpha}$. This proves the decomposition (3.1.1) above. For any $\alpha \in \Delta^{P}$, define an open subset

$$
X_{\alpha}:=G_{k} \backslash \bigcup_{\beta \in \Delta^{P}, \beta \neq \alpha} Z_{\beta} .
$$

Clearly, $U_{\mu} \subset X_{\alpha}$ and one has $X_{\alpha} \backslash U_{\mu}=E \cdot s_{\alpha}$. In particular, $X_{\alpha} \backslash U_{\mu}$ is irreducible. We define a morphism that satisfies the conditions of Definition 3.1.1 for the pair $\left(X_{\alpha}, U_{\mu}\right)$.

We take an isomorphism $u_{\alpha}: \mathbb{G}_{\mathrm{a}} \rightarrow U_{\alpha}$ for $\alpha \in \Phi$ so that $\left(u_{\alpha}\right)_{\alpha \in \Phi}$ is a realisation in the sense of [Spr98, §8.1.4]. In particular, we have

$$
t u_{\alpha}(x) t^{-1}=u_{\alpha}(\alpha(t) x)
$$

for $x \in \mathbb{G}_{\mathrm{a}}$ and $t \in T$. For $\alpha \in \Phi$, there is a unique homomorphism

$$
\phi_{\alpha}: \mathrm{SL}_{2, k} \rightarrow G_{k}
$$

such that

$$
\phi_{\alpha}\left(\left(\begin{array}{ll}
1 & x \\
0 & 1
\end{array}\right)\right)=u_{\alpha}(x), \quad \phi_{\alpha}\left(\left(\begin{array}{ll}
1 & 0 \\
x & 1
\end{array}\right)\right)=u_{-\alpha}(x)
$$


as in [Spr98, §9.2.2]. Also note that $\phi_{\alpha}\left(\operatorname{diag}\left(t, t^{-1}\right)\right)=\alpha^{\vee}(t)$.

Let $\alpha \in \Delta^{P}$. Set $Y=E \times \mathbb{A}^{1}$ and

$$
\psi_{\alpha}: Y \rightarrow G_{k} ;((x, y), t) \mapsto x \phi_{\alpha}(A(t)) y^{-1} \quad \text { where } A(t)=\left(\begin{array}{cc}
t & 1 \\
-1 & 0
\end{array}\right) \in \mathrm{SL}_{2, k}
$$

Note that $\phi_{\alpha}(A(0))=s_{\alpha}$ in $W$. The following identity will be crucial for later purposes:

$$
A(t)=\left(\begin{array}{cc}
1 & 0 \\
-t^{-1} & 1
\end{array}\right)\left(\begin{array}{cc}
t & 0 \\
0 & t^{-1}
\end{array}\right)\left(\begin{array}{cc}
1 & t^{-1} \\
0 & 1
\end{array}\right)
$$

Let $\wp: T \rightarrow T ; g \mapsto g \varphi(g)^{-1}$ be the Lang torsor. Then $\wp$ induces the isomorphism

$$
\wp_{*}: X_{*}(T)_{\mathbb{R}} \stackrel{\sim}{\longrightarrow} X_{*}(T)_{\mathbb{R}} ; \delta \mapsto \wp \circ \delta=\delta-q \sigma(\delta) .
$$

We put $\delta_{\alpha}=\wp_{*}^{-1}\left(\alpha^{\vee}\right)$. Recall that $\sigma$ denotes the $q$ th power Frobenius action on $\Delta$. We put

$$
m_{\alpha}=\min \left\{m \geq 1 \mid \sigma^{-m}(\alpha) \notin I\right\}
$$

and $t_{\alpha}=t^{-1} \alpha\left(\varphi\left(\delta_{\alpha}(t)\right)\right)^{-1}=t \alpha\left(\delta_{\alpha}(t)\right)^{-1} \in t^{\mathbb{Q}}$, where $t$ is an indeterminate.

Proposition 3.1.4. The following properties hold:

(1) The image of $\psi_{\alpha}$ is contained in $X_{\alpha}$.

(2) For any $(x, y) \in E$ and $t \in \mathbb{A}^{1}$, one has $\psi_{\alpha}((x, y), t) \in U_{\mu} \Longleftrightarrow t \neq 0$.

(3) For all $(x, y) \in E$, we have $\psi_{\alpha}((x, y), 0) \in E \cdot s_{\alpha}$.

Proof. It suffices to show (2) and (3). If $t=0$, we have $\phi_{\alpha}(A(0))=s_{\alpha}$ in $W$. Hence, $\psi_{\alpha}((x, y), 0) \in$ $E \cdot s_{\alpha}$. Assume that $t \neq 0$. We put

$$
u_{t, \alpha}=\prod_{i=1}^{m_{\alpha}-1} \phi_{\sigma^{-i}(\alpha)}\left(\left(\begin{array}{cc}
1 & -t_{\alpha}^{\frac{1}{q^{i}}} \\
0 & 1
\end{array}\right)\right),
$$

where the products are taken in the increasing order of indices. By (3.1.3) and the definitions of $\delta_{\alpha}, t_{\alpha}$ and $u_{t, \alpha}$, we have

$$
\begin{aligned}
\phi_{\alpha}(A(t)) & =\phi_{\alpha}\left(\left(\begin{array}{cc}
1 & 0 \\
-t^{-1} & 1
\end{array}\right)\right) \delta_{\alpha}(t) \varphi\left(\delta_{\alpha}(t)\right)^{-1} \phi_{\alpha}\left(\left(\begin{array}{cc}
1 & t^{-1} \\
0 & 1
\end{array}\right)\right) \\
& =\phi_{\alpha}\left(\left(\begin{array}{cc}
1 & 0 \\
-t^{-1} & 1
\end{array}\right)\right) \delta_{\alpha}(t) \phi_{\alpha}\left(\left(\begin{array}{cc}
1 & t_{\alpha} \\
0 & 1
\end{array}\right)\right) \varphi\left(\delta_{\alpha}(t)\right)^{-1} \\
& =\phi_{\alpha}\left(\left(\begin{array}{cc}
1 & 0 \\
-t^{-1} & 1
\end{array}\right)\right) \delta_{\alpha}(t) u_{t, \alpha}\left(\varphi\left(\delta_{\alpha}(t)\right) \phi_{\alpha}\left(\left(\begin{array}{cc}
1 & -t_{\alpha} \\
0 & 1
\end{array}\right)\right) u_{t, \alpha}\right)^{-1} .
\end{aligned}
$$

We have

$$
\left(\phi_{\alpha}\left(\left(\begin{array}{cc}
1 & 0 \\
-t^{-1} & 1
\end{array}\right)\right) \delta_{\alpha}(t) u_{t, \alpha}, \varphi\left(\delta_{\alpha}(t)\right) \phi_{\alpha}\left(\left(\begin{array}{cc}
1 & -t_{\alpha} \\
0 & 1
\end{array}\right)\right) u_{t, \alpha}\right) \in E
$$

because

$$
\phi_{\alpha}\left(\left(\begin{array}{cc}
1 & 0 \\
-t^{-1} & 1
\end{array}\right)\right) \in R_{\mathrm{u}}(P), \quad \phi_{\sigma^{-\left(m_{\alpha}-1\right)}(\alpha)}\left(\left(\begin{array}{cc}
1 & -t_{\alpha}^{\frac{1}{q^{m_{\alpha}-1}}} \\
0 & 1
\end{array}\right)\right) \in R_{\mathrm{u}}(Q)
$$

by $\alpha \notin I$ and $\sigma^{-\left(m_{\alpha}-1\right)}(\alpha) \notin \sigma(I)$. Hence, we have $\psi_{\alpha}((x, y), t) \in U_{\mu}$ if $t \neq 0$.

Set $Y_{0}:=E \times \mathbb{G}_{\mathrm{m}} \subset Y$. We obtain a $\operatorname{map} \psi_{\alpha}: Y_{0} \rightarrow U_{\mu}$. 
Corollary 3.1.5. Let $f: U_{\mu} \rightarrow \mathbb{A}^{n}$ be a regular map. Then $f$ extends to a regular map $G_{k} \rightarrow \mathbb{A}^{n}$ if and only if for all $\alpha \in \Delta^{P}$, the map $f \circ \psi_{\alpha}: Y_{0} \rightarrow \mathbb{A}^{n}$ extends to a map $Y \rightarrow \mathbb{A}^{n}$.

Proof. Applying Lemma 3.1.2 and Lemma 3.1.3 to the coordinate functions of $f$, we can extend $f$ to $\bigcup_{\alpha \in \Delta^{P}} X_{\alpha}$. Because the complement of $\bigcup_{\alpha \in \Delta^{P}} X_{\alpha}$ in $G$ has codimension $\geq 2$, we can extend $f$ to $G$ by normality.

\subsection{The space of $\mu$-ordinary sections}

Recall that $\mathcal{U}_{\mu}=\left[E \backslash U_{\mu}\right] \subset G$-Zip ${ }^{\mu}$ denotes the $\mu$-ordinary locus (see Subsection 2.2.4). The open substack $\mathcal{U}_{\mu} \subset G$-Zip ${ }^{\mu}$ is dense and hence induces an obvious injective map

$$
H^{0}\left(G-\operatorname{Zip}^{\mu}, \mathcal{V}(\rho)\right) \rightarrow H^{0}\left(\mathcal{U}_{\mu}, \mathcal{V}(\rho)\right)
$$

for any $(V, \rho) \in \operatorname{Rep}(P)$. This will give an upper bound approximation of the space $H^{0}\left(G\right.$-Zip $\left.{ }^{\mu}, \mathcal{V}(\rho)\right)$. We claim that $1 \in U_{\mu}$. Indeed, by Theorem 2.2.4, $U_{\mu}$ coincides with the $E$-orbit of the element $w_{0, I} w_{0} z^{-1}$. Because $z=\sigma\left(w_{0, I}\right) w_{0}$, we obtain $w_{0, I} w_{0} z^{-1}=w_{0, I} \sigma\left(w_{0, I}\right)$. This element is in the same $E$-orbit as 1 , because $\left(w_{0, I}, \sigma\left(w_{0, I}\right)\right) \in E$. This proves the claim.

We denote by $L_{\varphi} \subset E$ the scheme-theoretical stabiliser of the element 1 . Note that

$$
L_{\varphi}=E \cap\left\{(x, x) \mid x \in G_{k}\right\}
$$

is a 0 -dimensional algebraic group. In general it is nonsmooth. Denote by $L_{0} \subset L$ the largest algebraic subgroup defined over $\mathbb{F}_{q}$. In other words,

$$
L_{0}=\bigcap_{n \geq 0} L^{\left(q^{n}\right)}
$$

In view of (3.2.1), it is clear that the restriction of the first projection $E \rightarrow P$ induces a closed immersion $L_{\varphi} \rightarrow P$. Hence, we will identify $L_{\varphi}$ with its image and view it as a subgroup of $P$.

\section{Lemma 3.2.1 ([KW18, Lemma 3.2.1]).}

(1) One has $L_{\varphi} \subset L$.

(2) The group $L_{\varphi}$ can be written as a semidirect product

$$
L_{\varphi}=L_{\varphi}^{\circ} \rtimes L_{0}\left(\mathbb{F}_{q}\right)
$$

where $L_{\varphi}^{\circ}$ is the identity component of $L_{\varphi}$. Furthermore, $L_{\varphi}^{\circ}$ is a finite unipotent algebraic group.

(3) Assume that $P$ is defined over $\mathbb{F}_{q}$. Then $L_{0}=L$ and $L_{\varphi}=L\left(\mathbb{F}_{q}\right)$, viewed as a constant algebraic group.

Proposition 3.2.2. The stack $\mathcal{U}_{\mu}$ is isomorphic to $B\left(L_{\varphi}\right)=\left[1 / L_{\varphi}\right]$, the classifying stack of $L_{\varphi}$.

Proof. The action map $E \rightarrow U_{\mu}, e \mapsto e \cdot 1$ induces an isomorphism $E / L_{\varphi} \simeq U_{\mu}$. Hence, $\mathcal{U}_{\mu}=$ $\left[E \backslash U_{\mu}\right] \simeq\left[E \backslash\left(E / L_{\varphi}\right)\right] \simeq\left[1 / L_{\varphi}\right]$.

Corollary 3.2.3. The category of vector bundles on $\mathcal{U}_{\mu}$ is equivalent to the category $\operatorname{Rep}\left(L_{\varphi}\right)$ of representations of $L_{\varphi}$. Furthermore, for all $(V, \rho) \in \operatorname{Rep}\left(L_{\varphi}\right)$, the space of global sections of the attached vector bundle $\mathcal{V}(\rho)$ on $\mathcal{U}_{\mu}$ identifies with the space of $L_{\varphi}$-invariants of $V$ :

$$
H^{0}\left(\mathcal{U}_{\mu}, \mathcal{V}(\rho)\right)=V^{L_{\varphi}} .
$$

Furthermore, this identification is functorial in $(V, \rho)$.

The identity (3.2.2) can be seen as an isomorphism between two functors $\operatorname{Rep}\left(L_{\varphi}\right) \rightarrow \operatorname{Vec}_{k}$. The notation $V^{L_{\varphi}}$ for the space of invariants is to be understood in a scheme-theoretical way as the set of 
$v \in V$ such that for any $k$-algebra $R$, one has $\rho(x) v=v$ in $V \otimes_{k} R$ for all $x \in L_{\varphi}(R)$. In particular, if $(V, \rho) \in \operatorname{Rep}(P)$ and $\mathcal{V}(\rho)$ is the attached vector bundle on $G$-Zip ${ }^{\mu}$, the restriction of $\mathcal{V}(\rho)$ to $\mathcal{U}_{\mu}$ is attached to the restriction of $\rho$ to $L_{\varphi}$, and the formula (3.2.2) applies similarly.

By (2.4.1), any $f \in V^{L_{\varphi}}=H^{0}\left(\mathcal{U}_{\mu}, \mathcal{V}(\rho)\right)$ corresponds bijectively to a unique function

$$
\tilde{f}: U_{\mu} \rightarrow V
$$

satisfying $\tilde{f}(1)=f$ and $\tilde{f}\left(a x b^{-1}\right)=\rho(a) \tilde{f}(x)$ for all $(a, b) \in E$ and all $x \in U_{\mu}$. The strategy to determine the space $H^{0}\left(G-\right.$ Zip $\left.^{\mu}, \mathcal{V}(\rho)\right)$ will be to characterise which of these functions extends to a function $G_{k} \rightarrow V$. We will use Corollary 3.1.5 for this purpose. As another preliminary, we introduce (a generalisation of) the Brylinski-Kostant filtration in the next section.

\subsection{Brylinski-Kostant filtration}

Lemma 3.3.1. Let $\alpha \in \Phi$. Let $V$ be a finite-dimensional algebraic representation of $T U_{\alpha}$. Let $v \in V_{v}$ for $v \in X^{*}(T)$. Then we have

$$
u_{\alpha}(x)(v)-v=\sum_{j=1}^{\infty} x^{j} v_{j}
$$

where $v_{j} \in V_{v+j \alpha}$.

Proof. This is proved in the proof of [Don85, Proposition 3.3.2]. We recall the argument. We write $u_{\alpha}(x) v$ as $\sum_{j \geq 0} x^{j} v_{j}$ for some $v_{j} \in V$. We note that $v_{0}=v$. By (3.1.2), we have $v_{j} \in V_{v+j \alpha}$.

For $\alpha \in \Phi$, we define $E_{\alpha}^{(j)}: V \rightarrow V$ by

$$
u_{\alpha}(x) v=\sum_{j \geq 0} x^{j} E_{\alpha}^{(j)}(v)
$$

for $j \geq 0$ and put $E_{\alpha}^{(j)}=0$ if $j<0$. By Lemma 3.3.1, we have $E_{\alpha}^{(j)}(v) \in V_{v+j \alpha}$ for $v \in V_{v}$.

Let $\Xi=\left(\alpha_{1}, \ldots, \alpha_{m}\right) \in \Phi^{m}$. Let $H$ be a closed subgroup scheme of $G$ contaning $T$ and $U_{\alpha_{i}}$ for $1 \leq i \leq m$. Let $V$ be a finite-dimensional algebraic representation of $H$. Let $\mathbf{a}=\left(a_{1}, \ldots, a_{m}\right) \in\left(k^{\times}\right)^{m}$ and $\mathbf{r}=\left(r_{1}, \ldots, r_{m}\right) \in \mathbb{R}^{m}$. We put

$$
\begin{aligned}
\left(\mathbb{Z}^{m}\right)_{\mathbf{r}} & =\left\{\left(n_{1}, \ldots, n_{m}\right) \in \mathbb{Z}^{m} \mid \sum_{i=1}^{m} n_{i} r_{i}=0\right\}, \\
\Lambda_{\Xi, \mathbf{r}} & =\left\{\sum_{i=1}^{m} n_{i} \alpha_{i} \mid\left(n_{1}, \ldots, n_{m}\right) \in\left(\mathbb{Z}^{m}\right)_{\mathbf{r}}\right\} .
\end{aligned}
$$

For $[v] \in X^{*}(T) / \Lambda_{\Xi, \mathbf{r}}$, we put

$$
V_{[v]}=\bigoplus_{v \in[v]} V_{v} .
$$

We use the notation $\mathbf{j}$ for $\left(j_{1}, \ldots, j_{m}\right) \in \mathbb{Z}^{m}$. For $[\mathbf{j}] \in \mathbb{Z}^{m} /\left(\mathbb{Z}^{m}\right)_{\mathbf{r}}$ and $[v] \in X^{*}(T) / \Lambda_{\Xi, \mathbf{r}}$, we put

$$
\begin{aligned}
{[\mathbf{j}] \cdot \mathbf{r} } & =\sum_{i=1}^{m} j_{i} r_{i} \in \mathbb{R}, \\
{[v]+[\mathbf{j}] \cdot \Xi } & =\left[v+\sum_{i=1}^{m} j_{i} \alpha_{i}\right] \in X^{*}(T) / \Lambda_{\Xi, \mathbf{r}},
\end{aligned}
$$


which are well defined. For $[v] \in X^{*}(T) / \Lambda_{\Xi, \mathbf{r}}$ and a function $\delta: X^{*}(T) \rightarrow \mathbb{R}$, we define Fil $_{\delta}^{\Xi, \mathbf{a}, \mathbf{r}} V_{[v]}$ by

$$
\bigcap_{[\mathbf{j}] \in \mathbb{Z}^{m} /\left(\mathbb{Z}^{m}\right) \mathbf{r} \mathbf{r}} \bigcap_{\substack{\chi \in[v]+[\mathbf{j}] \cdot \Xi,[\mathbf{j}] \cdot \mathbf{r}>\delta(\chi)}} \operatorname{Ker}\left(\sum_{\mathbf{j} \in[\mathbf{j}]} \operatorname{pr}_{\chi} \circ a_{1}^{j_{1}} E_{\alpha_{1}}^{\left(j_{1}\right)} \circ \cdots \circ a_{m}^{j_{m}} E_{\alpha_{m}}^{\left(j_{m}\right)}: V_{[v]} \rightarrow V_{\chi}\right),
$$

where $\operatorname{pr}_{\chi}: V_{[v]+[\mathbf{j}] \cdot \Xi} \rightarrow V_{\chi}$ denotes the projection.

Example 3.3.2. Assume that $\Xi=(\alpha) \in \Phi, r_{1}=1$ and $\delta$ is a constant function $c \in \mathbb{R}$. Then $\Lambda_{\Xi, \mathbf{r}}=0$ and $V_{[v]}=V_{v}$ for $v \in X^{*}(T)$. In this case,

$$
\operatorname{Fil}_{c}^{\Xi, \mathbf{a}, \mathbf{r}} V_{v}=\bigcap_{j>c} \operatorname{Ker}\left(E_{\alpha}^{(j)}: V_{v} \rightarrow V_{v+j \alpha}\right),
$$

which we simply write $\mathrm{Fil}_{c}^{\alpha} V_{v}$. This is a Brylinski-Kostant filtration (cf. [XZ19, (3.3.2)]).

\subsection{Main result}

We now investigate the space of global sections over $G$-Zip ${ }^{\mu}$ of the vector bundle $\mathcal{V}(\rho)$ for $(V, \rho) \in$ $\operatorname{Rep}(P)$. By (3.2.2), this space is contained in $V^{L_{\varphi}}$. Conversely, the problem is to determine which $f \in V^{L_{\varphi}}$ correspond to sections of $\mathcal{V}(\rho)$ that extend from $\mathcal{U}_{\mu}$ to $G$-Zip ${ }^{\mu}$. Equivalently, we ask for which $f \in V^{L_{\varphi}}$ the regular function $\tilde{f}: U_{\mu} \rightarrow V$ defined in (3.2.3) extends to a regular function $G_{k} \rightarrow V$.

Recall the definition of the integer $m_{\alpha}$ in (3.1.4) for each $\alpha \in \Delta^{P}$. For example, if $P$ is defined over $\mathbb{F}_{q}$, then $m_{\alpha}=1$ for all $\alpha \in \Delta^{P}$. We put $\mathbf{a}_{\alpha}=(-1, \ldots,-1) \in\left(k^{\times}\right)^{m_{\alpha}}$. For $\alpha \in \Delta^{P}$, we put $\Xi_{\alpha}=\left(-\alpha, \sigma^{-1}(\alpha), \ldots, \sigma^{-\left(m_{\alpha}-1\right)}(\alpha)\right)$ and $\mathbf{r}_{\alpha}=\left(r_{\alpha, 1}, \ldots, r_{\alpha, m_{\alpha}}\right)$, where $r_{\alpha, 1}=1-\left\langle\alpha, \delta_{\alpha}\right\rangle$ and

$$
r_{\alpha, i}=\frac{\left\langle\alpha, \delta_{\alpha}\right\rangle-1}{q^{i-1}}
$$

for $2 \leq i \leq m_{\alpha}$. We view $\delta_{\alpha}$ as a function $X^{*}(T) \rightarrow \mathbb{R}$ by $\chi \mapsto\left\langle\chi, \delta_{\alpha}\right\rangle$.

Theorem 3.4.1. Let $(V, \rho) \in \operatorname{Rep}(P)$. Via the inclusion $H^{0}\left(G-\operatorname{Zip}^{\mu}, \mathcal{V}(\rho)\right) \subset V^{L_{\varphi}}$ (see Corollary 3.2.3) one has an identification

$$
H^{0}\left(G-\operatorname{Zip}^{\mu}, \mathcal{V}(\rho)\right)=V^{L_{\varphi}} \cap \bigcap_{\alpha \in \Delta^{P}} \bigoplus_{[v] \in X^{*}(T) / \Lambda_{\Xi_{\alpha}, \mathbf{r}_{\alpha}}} \operatorname{Fil}_{\delta_{\alpha}}^{\Xi_{\alpha}, \boldsymbol{a}_{\alpha}, \mathbf{r}_{\alpha}} V_{[v]} .
$$

Proof. Let $f \in V^{L_{\varphi}}$, and let $\tilde{f}: U_{\mu} \rightarrow V$ be the function defined in (3.2.3). It suffices to show that $\tilde{f}$ extends to $G$ if and only if

$$
f \in \bigoplus_{[v] \in X^{*}(T) / \Lambda_{\Xi_{\alpha}, \mathbf{r}_{\alpha}}} \operatorname{Fil}_{\delta_{\alpha}}^{\Xi_{\alpha}, \mathbf{a}_{\alpha}, \mathbf{r}_{\alpha}} V_{[v]}
$$

for all $\alpha \in \Delta^{P}$. By Corollary 3.1.5, $\tilde{f}$ extends to $G_{k}$ if and only if $\tilde{f} \circ \psi_{\alpha}: Y_{0} \rightarrow V$ extends to a function $Y \rightarrow V$. We now give an explicit formula for $\tilde{f} \circ \psi_{\alpha}((x, y), t)$. Using (3.1.5) and (3.1.6), the element $\psi_{\alpha}((x, y), t) \in U$ can be written as $x_{1} x_{2}^{-1}$ with $\left(x_{1}, x_{2}\right) \in E$ and

$$
x_{1}=x \phi_{\alpha}\left(\left(\begin{array}{cc}
1 & 0 \\
-t^{-1} & 1
\end{array}\right)\right) \delta_{\alpha}(t) u_{t, \alpha}, \quad x_{2}=y \varphi\left(\delta_{\alpha}(t)\right) \phi_{\alpha}\left(\left(\begin{array}{cc}
1 & -t_{\alpha} \\
0 & 1
\end{array}\right)\right) u_{t, \alpha} .
$$

It follows that

$$
\left(\tilde{f} \circ \psi_{\alpha}\right)((x, y), t)=\tilde{f}\left(x_{1} x_{2}^{-1}\right)=\rho\left(x_{1}\right) f=\rho(x) \rho\left(\phi_{\alpha}\left(\left(\begin{array}{cc}
1 & 0 \\
-t^{-1} & 1
\end{array}\right)\right) \delta_{\alpha}(t) u_{t, \alpha}\right) f .
$$


Hence, the function $\tilde{f} \circ \psi_{\alpha}$ extends to $Y$ if and only if the function

$$
F_{\alpha}: t \mapsto \rho\left(\phi_{\alpha}\left(\left(\begin{array}{cc}
1 & 0 \\
-t^{-1} & 1
\end{array}\right)\right) \delta_{\alpha}(t) u_{t, \alpha}\right) f
$$

lies in $k[t] \otimes V$. Write $f=\sum_{v \in X^{*}(T)} f_{V}$ by the weight decomposition of $f$. We put

$$
f_{v, \Xi_{\alpha}}^{\mathbf{j}}=E_{-\alpha}^{\left(j_{1}\right)} E_{\sigma^{-1}(\alpha)}^{\left(j_{2}\right)} \cdots E_{\sigma^{-\left(m_{\alpha}-1\right)}(\alpha)}^{\left(j_{m_{\alpha}}\right)} f_{v} \in V_{v+\mathbf{j} \cdot \Xi_{\alpha}}
$$

for $\mathbf{j}=\left(j_{1}, \ldots, j_{m_{\alpha}}\right) \in \mathbb{Z}^{m_{\alpha}}$ and $v \in X^{*}(T)$. We obtain

$$
\begin{aligned}
F_{\alpha}(t) & =\rho\left(\delta_{\alpha}(t) \phi_{\alpha}\left(\left(\begin{array}{cc}
1 & 0 \\
-\alpha\left(\delta_{\alpha}(t)\right) t^{-1} & 1
\end{array}\right)\right) u_{t, \alpha}\right) f \\
& =\sum_{v} \rho\left(\delta_{\alpha}(t) \phi_{\alpha}\left(\left(\begin{array}{cc}
1 & 0 \\
-t^{\left\langle\alpha, \delta_{\alpha}\right\rangle-1} & 1
\end{array}\right)\right) \prod_{i=2}^{m_{\alpha}} \phi_{\sigma^{-(i-1)}(\alpha)}\left(\left(\begin{array}{cc}
1 & -t_{\alpha}^{\frac{1}{q^{i-1}}} \\
0 & 1
\end{array}\right)\right)\right) f_{v} \\
& =\sum_{v} \rho\left(\delta_{\alpha}(t)\right) \sum_{\mathbf{j} \in \mathbb{Z}^{m_{\alpha}}}\left(\left(-t^{\left\langle\alpha, \delta_{\alpha}\right\rangle-1}\right)^{j_{1}} \prod_{i=2}^{m_{\alpha}}\left(-t_{\alpha}^{\frac{1}{q^{i-1}}}\right)^{j_{i}}\right) f_{v, \Xi_{\alpha}}^{\mathbf{j}} \\
& =\sum_{v} \sum_{\mathbf{j} \in \mathbb{Z}^{m_{\alpha}}} t^{\left\langle v+\mathbf{j} \cdot \Xi_{\alpha}, \delta_{\alpha}\right\rangle}\left(\left(-t^{\left\langle\alpha, \delta_{\alpha}\right\rangle-1}\right)^{j_{1}} \prod_{i=2}^{m_{\alpha}}\left(-t_{\alpha}^{\frac{1}{q^{i-1}}}\right)^{j_{i}}\right) f_{v, \Xi_{\alpha}}^{\mathbf{j}} .
\end{aligned}
$$

For fixed $\chi \in X^{*}(T)$, let $F_{\alpha, \chi}(t)$ be the $V_{\chi}$-component of $F_{\alpha}(t)$. Then we have

$$
\begin{aligned}
& F_{\alpha, \chi}(t)=\sum_{\mathbf{j} \in \mathbb{Z}^{m_{\alpha}}} t^{\left\langle\chi, \delta_{\alpha}\right\rangle}\left(\left(-t^{\left\langle\alpha, \delta_{\alpha}\right\rangle-1}\right)^{j_{1}} \prod_{i=2}^{m_{\alpha}}\left(-t_{\alpha}^{\frac{1}{q^{i-1}}}\right)^{j_{i}}\right) f_{\mathcal{\chi}-\mathbf{j} \cdot \Xi_{\alpha}, \Xi_{\alpha}}^{\mathbf{j}} \\
& =\sum_{[\mathbf{j}] \in \mathbb{Z}^{m_{\alpha}}\left(\mathbb{Z}^{m_{\alpha}}\right)_{\mathbf{r}_{\alpha}}} \sum_{\mathbf{j} \in[\mathbf{j}]} t^{\left\langle\chi, \delta_{\alpha}\right\rangle-\mathbf{j} \cdot \mathbf{r}_{\alpha}}(-1)^{\sum_{i=1}^{m_{\alpha}} j_{i}} f_{\chi-\mathbf{j} \cdot \Xi_{\alpha}, \Xi_{\alpha}}^{\mathbf{j}} .
\end{aligned}
$$

The exponents of $t$ in two terms in the last expression are equal if and only if the indices belong to the same coset in $\mathbb{Z}^{m_{\alpha}} /\left(\mathbb{Z}^{m_{\alpha}}\right)_{\mathbf{r}_{\alpha}}$. Therefore, $F_{\alpha, \chi}(t)$ lies in $k[t] \otimes V_{\chi}$ for all $\chi \in X^{*}(T)$ if and only if we have

$$
\sum_{\mathbf{j} \in[\mathbf{j}]}(-1)^{\sum_{i=1}^{m_{\alpha}} j_{i}} f_{\chi-\mathbf{j} \cdot \Xi_{\alpha}, \Xi_{\alpha}}^{\mathbf{j}}=0
$$

for all $\chi \in X^{*}(T)$ and $[\mathbf{j}] \in \mathbb{Z}^{m_{\alpha}} /\left(\mathbb{Z}^{m_{\alpha}}\right)_{\mathbf{r}_{\alpha}}$ such that $\left.\mathbf{j} \cdot \mathbf{r}_{\alpha}\right\rangle\left\langle\chi, \delta_{\alpha}\right\rangle$. This condition is equivalent to that $f$ belongs to $\bigoplus_{[v] \in X^{*}(T) / \Lambda_{\Xi_{\alpha}, \mathbf{r}_{\alpha}}} \mathrm{Fil}_{\delta_{\alpha}}^{\Xi_{\alpha}, \mathbf{a}_{\alpha}, \mathbf{r}_{\alpha}} V_{[v]}$. Hence, the claim follows.

We now give some corollaries of Theorem 3.4.1 in that case where the formula (3.4.1) becomes simpler. For $v \in X^{*}(T)$ and $\chi \in X^{*}(T)_{\mathbb{R}}$, we put

$$
\operatorname{Fil}_{\chi}^{P} V_{v}=\bigcap_{\alpha \in \Delta^{P}} \operatorname{Fil}_{\left\langle\chi, \alpha^{\vee}\right\rangle}^{-\alpha} V_{v}
$$

where $\mathrm{Fil}_{\left\langle\chi, \alpha^{\vee}\right\rangle}^{-\alpha} V_{v}$ was defined in Example 3.3.2. The morphism $\wp: T \rightarrow T$ induces the isomorphism

$$
\wp^{*}: X^{*}(T)_{\mathbb{R}} \stackrel{\sim}{\longrightarrow} X^{*}(T)_{\mathbb{R}} ; \lambda \mapsto \lambda \circ \wp=\lambda-q \sigma^{-1}(\lambda) .
$$


Corollary 3.4.2. Assume that $P$ is defined over $\mathbb{F}_{q}$. Let $(V, \rho) \in \operatorname{Rep}(P)$. Via the inclusion $H^{0}\left(G-\operatorname{Zip}^{\mu}, \mathcal{V}(\rho)\right) \subset V^{L\left(\mathbb{F}_{q}\right)}$ one has

$$
H^{0}\left(G-\operatorname{Zip}^{\mu}, \mathcal{V}(\rho)\right)=V^{L\left(\mathbb{F}_{q}\right)} \cap \bigoplus_{\nu \in X^{*}(T)} \operatorname{Fil}_{\wp^{*-1}(v)}^{P} V_{\nu}
$$

Proof. For $\alpha \in \Delta^{P}$ and $v \in X^{*}(T)$, we have

$$
\operatorname{Fil}_{\delta_{\alpha}}^{\Xi_{\alpha}, \mathbf{a}_{\alpha}, \mathbf{r}_{\alpha}} V_{[v]}=\operatorname{Fil}_{\left\langle v, \delta_{\alpha}\right\rangle}^{-\alpha} V_{v}=\mathrm{Fil}_{\left\langle\wp^{*-1}(v), \alpha^{\vee}\right\rangle}^{-\alpha} V_{v} .
$$

Hence, the claim follows from Lemma 3.2.1(3) and Theorem 3.4.1.

Assume again that $P$ is defined over $\mathbb{F}_{q}$. To simplify further, assume that $(V, \rho) \in \operatorname{Rep}(P)$ is trivial on the unipotent radical $R_{\mathrm{u}}(P)$. Then we have $E_{-\alpha}^{(j)}=0$ for all $\alpha \in \Delta^{P}$ and all $j>0$. It follows that $\mathrm{Fil}_{c}^{-\alpha} V_{v}=V_{v}$ for $c \geq 0$ and $\mathrm{Fil}_{c}^{-\alpha} V_{v}=0$ for $c<0$. We obtain that for all $\chi \in X^{*}(T)_{\mathbb{R}}$, one has

$$
\operatorname{Fil}_{\chi}^{P} V_{v}= \begin{cases}V_{v} & \text { if for all } \alpha \in \Delta^{P} \text { one has }\left\langle\chi, \alpha^{\vee}\right\rangle \geq 0, \\ 0 & \text { otherwise. }\end{cases}
$$

Define a subspace $V_{\geq 0}^{\Delta^{P}} \subset V$ as follows:

$$
V_{\geq 0}^{\Delta^{P}}=\bigoplus_{\left\langle v, \delta_{\alpha}\right\rangle \geq 0, \forall \alpha \in \Delta^{P}} V_{v} .
$$

For example, if $T$ is split over $\mathbb{F}_{q}$, then $\delta_{\alpha}=-\alpha^{\vee} /(q-1)$, and therefore $V_{\geq 0}^{\Delta^{P}}$ is the direct sum of the weight spaces $V_{v}$ for those $v \in X^{*}(T)$ satisfying $\left\langle v, \alpha^{\vee}\right\rangle \leq 0$ for all $\alpha \in \Delta^{P}$.

Corollary 3.4.3. Assume that $P$ is defined over $\mathbb{F}_{q}$ and furthermore that $(V, \rho) \in \operatorname{Rep}(P)$ is trivial on the unipotent radical $R_{u}(P)$. Then one has an equality

$$
H^{0}\left(G-\operatorname{Zip}^{\mu}, \mathcal{V}(\rho)\right)=V^{L\left(\mathbb{F}_{q}\right)} \cap V_{\geq 0}^{\Delta^{P}} .
$$

This formula recovers the result [Kos19, Theorem 1] (with slightly different notation). In [Kos19, Theorem 1], only the special case when $G$ is split over $\mathbb{F}_{p}$ and $V$ is of the form $V_{I}(\lambda)$ was considered.

\subsection{Perfection}

As noted in Remark 2.5.2, the perfection of the stack of $G$-zips appears in connection with the moduli of local shtukas. In [XZ17, Lemma 5.3.6], the zip datum that appears satisfies that $P$ is defined over $\mathbb{F}_{q}$. We do not make this assumption here. For a scheme $X$ over $k$, define the perfection of $X$ as the projective limit

$$
X^{\mathrm{pf}}:={\underset{\iota}{\varphi_{X}}}_{\lim } X
$$

where $\varphi_{X}$ denotes the absolute $q$ th power Frobenius endomorphism of $X$. There is a natural map $X^{\mathrm{pf}} \rightarrow X$. We have an isomorphism

$$
X^{\mathrm{pf}} \simeq \lim _{\longleftarrow}\left(\cdots \stackrel{\varphi}{\rightarrow} X^{\left(q^{-2}\right)} \stackrel{\varphi}{\rightarrow} X^{\left(q^{-1}\right)} \stackrel{\varphi}{\rightarrow} X\right)
$$

where $\varphi$ denotes the relative $q$ th power Frobenius endomorphism. The perfection of $G$-Zip ${ }^{\mu}$ is then given by

$$
G-Z i p^{\mu, \mathrm{pf}}=\left[E^{\mathrm{pf}} \backslash G_{k}^{\mathrm{pf}}\right] .
$$


Similar to Proposition 3.2.2, the perfection of the $\mu$-ordinary locus $\mathcal{U}_{\mu}^{\mathrm{pf}}$ is isomorphic to $\left[1 / L_{\varphi}^{\mathrm{pf}}\right]$. Because $L_{\varphi}=L_{\varphi}^{\circ} \rtimes L_{0}\left(\mathbb{F}_{q}\right)$ by Lemma 3.2.1(2), we obtain

$$
\mathcal{U}_{\mu}^{\mathrm{pf}}=\left[1 / L_{0}\left(\mathbb{F}_{q}\right)\right]
$$

If $(V, \rho)$ is a $P$-representation, then we obtain a $P^{\text {pf }}$-representation by pullback, which we denote by $\rho^{\mathrm{pf}}$. This yields a vector bundle $\mathcal{V}\left(\rho^{\mathrm{pf}}\right)$ on $G$-Zip ${ }^{\mu, \mathrm{pf}}$, which also coincides with the pullback of $\mathcal{V}(\rho)$ under the natural map

$$
G-\mathrm{Zip}^{\mu, \mathrm{pf}} \rightarrow G-\mathrm{Zip}^{\mu}
$$

By equation (3.5.1), we see that the space $H^{0}\left(G-\operatorname{Zip}^{\mu, \mathrm{pf}}, \mathcal{V}\left(\rho^{\mathrm{pf}}\right)\right)$ is naturally a subspace of $V^{L_{0}\left(\mathbb{F}_{q}\right)}$.

Corollary 3.5.1. Let $(V, \rho) \in \operatorname{Rep}(P)$. We have

$$
H^{0}\left(G-Z_{i p}^{\mu, p f}, \mathcal{V}\left(\rho^{\mathrm{pf}}\right)\right)=V^{L_{0}\left(\mathbb{F}_{q}\right)} \cap \bigcap_{\alpha \in \Delta^{P}[v] \in X^{*}(T) / \Lambda_{\Xi_{\alpha}, \mathbf{r}_{\alpha}}} \operatorname{Fil}_{\delta_{\alpha}}^{\Xi_{\alpha}, \boldsymbol{a}_{\alpha}, \mathbf{r}_{\alpha}} V_{[v]}
$$

Proof. Let $d$ be the smallest positive integer such that $\mu$ is defined over $\mathbb{F}_{q d}$. We show that $H^{0}\left(G\right.$-Zip $\left.{ }^{\mu \text {,pf }}, \mathcal{V}\left(\rho^{\mathrm{pf}}\right)\right)$ is given by the subspace of elements $f \in V$ such that there exists $n \geq 1$ with $f \in H^{0}\left(G\right.$-Zip $\left.{ }^{\mu}, \mathcal{V}\left(\rho^{\left(q^{n d}\right)}\right)\right)$. Indeed, such a section is given by a map $f: G_{k}^{\mathrm{pf}} \rightarrow V$ satisfying an $E^{\mathrm{pf}}-$ equivariance condition with respect to $\rho^{\mathrm{pf}}$. Because $V$ is a scheme of finite type, such a map is given by a map $f_{n}: G_{k} \rightarrow V$ at a finite level of the system $\left(\cdots \stackrel{\varphi^{d}}{\longrightarrow} G_{k} \stackrel{\varphi^{d}}{\longrightarrow} G_{k}\right)$. We have

$$
\operatorname{Fil}_{\delta_{\alpha}}^{\Xi_{\alpha}, \mathbf{a}_{\alpha}, \mathbf{r}_{\alpha}} V_{\left[q^{n d} v\right]}^{\left(q^{n d}\right)}=\operatorname{Fil}_{\delta_{\alpha}}^{\Xi_{\alpha}, \mathbf{a}_{\alpha}, \mathbf{r}_{\alpha}} V_{[v]}
$$

Hence, changing $\rho$ to $\rho^{\left(q^{n}\right)}$ only affects $V^{L_{\varphi}}$. The result follows.

\subsection{L-semisimplification}

If $\rho: P \rightarrow \mathrm{GL}(V)$ is an arbitrary representation, we can attach a $P$-representation $\left(V, \rho^{L \text {-ss }}\right)$ that is trivial on $R_{\mathrm{u}}(P)$. The representation $\rho^{L \text {-ss }}$ is defined as the composition

$$
\rho^{L \text {-ss }}: P \stackrel{\theta_{L}^{P}}{\longrightarrow} L \stackrel{\rho}{\rightarrow} \mathrm{GL}(V)
$$

where $\theta_{L}^{P}: P \rightarrow L$ is the natural projection map whose kernel is $R_{\mathrm{u}}(P)$, as defined in Subsection 2.2.1. We call $\rho^{L \text {-ss }}$ the $L$-semisimplification of $\rho$. We sometimes write $V^{L \text {-ss }}$ to denote this representation (even though the underlying vector space is the same as $V$ ).

One obvious property of $V^{L \text {-ss }}$ is $\left(V^{L \text {-ss }}\right)^{L_{\varphi}}=V^{L_{\varphi}}$ because $L_{\varphi} \subset L$ by Lemma 3.2.1(1). In particular, by Corollary 3.2.3, we have for all $(V, \rho) \in \operatorname{Rep}(P)$ the equality

$$
H^{0}\left(\mathcal{U}_{\mu}, \mathcal{V}\left(\rho^{L-s s}\right)\right)=H^{0}\left(\mathcal{U}_{\mu}, \mathcal{V}(\rho)\right)
$$

Note that this identification is somewhat indirect: It is not induced by a morphism between the sheaves $\mathcal{V}(\rho)$ and $\mathcal{V}\left(\rho^{L \text {-ss }}\right)$. For $f \in H^{0}\left(\mathcal{U}_{\mu}, \mathcal{V}(\rho)\right)$, we will write $f^{L \text {-ss }}$ for its image under the identification (3.6.1) and call it the $L$-semisimplification of $f$. As an element of $V, f^{L \text {-ss }}$ is the same as $f$, but we want to emphasise the fact that the representation has changed.

We now give another interpretation of $L$-semisimplification when $P$ is defined over $\mathbb{F}_{q}$. Write again $U_{\mu} \subset G_{k}$ for the unique open $E$-orbit and recall that $1 \in U_{\mu}$ (see Subsection 3.2). 
Lemma 3.6.1. Assume that $P$ is defined over $\mathbb{F}_{q}$. There exists a unique regular map $\Theta: U_{\mu} \rightarrow L$ such that for any $(a, b) \in E$, one has

$$
\Theta\left(a b^{-1}\right)=\theta_{L}^{P}(a) \theta_{L}^{Q}(b)^{-1} .
$$

Furthermore, we have $L \subset U_{\mu}$ and the inclusion $L \subset U_{\mu}$ is a section of $\Theta$.

Proof. First, note that because $P$ is defined over $\mathbb{F}_{q}$, one has $L=M$; hence, the formula (3.6.2) makes sense. The unicity of $\Theta$ is obvious. For the existence, consider the map $\tilde{\Theta}: E \rightarrow L ;(a, b) \mapsto$ $\theta_{L}^{P}(a) \theta_{L}^{Q}(b)^{-1}$. Because $P$ is defined over $\mathbb{F}_{q}$, one has $L_{\varphi}=L\left(\mathbb{F}_{q}\right)$ (Lemma 3.2.13). For all $(a, b) \in E$ and all $x \in L\left(\mathbb{F}_{q}\right)$, one has $\tilde{\Theta}(a x, b x)=\tilde{\Theta}(a, b)$. Hence, $\tilde{\Theta}$ factors to a map $\Theta: E / L\left(\mathbb{F}_{q}\right) \simeq U_{\mu} \rightarrow L$. This proves the first result. Now, if $x \in L$, we can write $x=a \varphi(a)^{-1}$ with $a \in L$ by Lang's theorem. Hence, $x \in U_{\mu}$ and $\Theta(x)=a \varphi(a)^{-1}=x$, so the second statement is proved.

Example 3.6.2. Consider the case $G=\operatorname{Sp}(2 n)_{\mathbb{F}_{q}}$ for $n \geq 1$. We write an element of $G_{k}$ as

$$
\left(\begin{array}{ll}
A & B \\
C & D
\end{array}\right)
$$

with $A, B, C, D$ square matrices of size $n \times n$. Let $P \subset G_{k}$ be the parabolic subgroup defined by the condition $B=0$ and $Q \subset G_{k}$ the parabolic subgroup defined by the condition $C=0$. We put $L=P \cap Q$. This gives a zip datum $(G, P, L, Q, L, \varphi)$. The Zariski open subset $U_{\mu} \subset G_{k}$ is the set of matrices in $G_{k}$ for which $A$ is invertible. The map $\Theta: U_{\mu} \rightarrow L$ is given by

$$
\Theta:\left(\begin{array}{ll}
A & B \\
C & D
\end{array}\right) \mapsto\left(\begin{array}{cc}
A & 0 \\
0 & D-C A^{-1} B
\end{array}\right) .
$$

Proposition 3.6.3. Assume that $P$ is defined over $\mathbb{F}_{q}$. Let $(V, \rho) \in \operatorname{Rep}(P)$ and let $f \in V^{L\left(\mathbb{F}_{q}\right)}$. Let $\tilde{f}$ be the corresponding function $U_{\mu} \rightarrow V$ defined in (3.2.3). Then the function $\widetilde{f^{L-s S}}: U_{\mu} \rightarrow V$ that corresponds to the L-semisimplification $f^{L-s s}$ is the composition

$$
U_{\mu} \stackrel{\Theta}{\longrightarrow} L \hookrightarrow U_{\mu} \stackrel{\tilde{f}}{\longrightarrow} V .
$$

Proof. Put $f^{\prime}=\tilde{f} \circ \Theta$. For $(a, b) \in E$ and $g \in U_{\mu}$ such that $g=a b^{-1}$, we have

$$
\begin{aligned}
f^{\prime}(g) & =f^{\prime}\left(a b^{-1}\right)=\tilde{f}\left(\Theta\left(a b^{-1}\right)\right) \\
& =\tilde{f}\left(\theta_{L}^{P}(a) \theta_{L}^{Q}(b)^{-1}\right)=\rho\left(\theta_{L}^{P}(a)\right) f=\rho^{L \text {-ss }}(a) f=\widetilde{f^{L-s s}}(g) .
\end{aligned}
$$

Hence, $f^{\prime}=\widetilde{f^{L-s s}}$.

Let $f \in H^{0}\left(G\right.$-Zip $\left.{ }^{\mu}, \mathcal{V}(\rho)\right)$ be a global section. We may view its restriction $\left.f\right|_{\mathcal{U}_{\mu}}$ as a section of $\mathcal{V}\left(\rho^{L \text {-ss }}\right)$ over $\mathcal{U}_{\mu}$ by the identification (3.6.1). It is thus natural to ask whether $\left(\left.f\right|_{\mathcal{U}_{\mu}}\right)^{L \text {-ss }}$ extends to a global section over $G$-Zip ${ }^{\mu}$. We prove that this holds when $P$ is defined over $\mathbb{F}_{q}$ in the following proposition.

Proposition 3.6.4. Assume that $P$ is defined over $\mathbb{F}_{q}$. The identification (3.6.1) extends to a commutative diagram

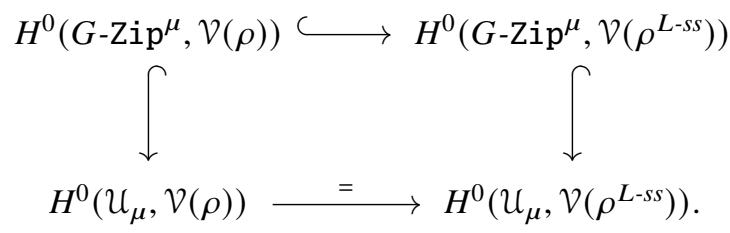


Proof. Let $f \in H^{0}\left(G\right.$-Zip $\left.{ }^{\mu}, \mathcal{V}(\rho)\right)$. Because $P$ is defined over $\mathbb{F}_{q}$, we can apply Corollary 3.4 .2 to the representation $(V, \rho)$. Furthermore, because $R_{\mathrm{u}}(P)$ acts trivially on $\left(V^{L \text {-ss }}, \rho^{L \text {-ss }}\right)$, we can apply Corollary 3.4.3 to $\left(V^{L \text {-ss }}, \rho^{L \text {-ss }}\right)$. Therefore, it suffices to show that for each $v \in X^{*}(T)$,

$$
V^{L\left(\mathbb{F}_{q}\right)} \cap \bigoplus_{v \in X^{*}(T)} \operatorname{Fil}_{\wp^{*-1}(v)}^{P} V_{v} \subset V^{L\left(\mathbb{F}_{q}\right)} \cap V_{\geq 0}^{\Delta^{P}} .
$$

By (3.4.3), it suffices to show the following: For any fixed $v \in X^{*}(T)$, if $\operatorname{Fil}_{\wp^{*-1}(v)}^{P} V_{v} \neq 0$, then $\left\langle\wp^{*-1}(v), \alpha^{\vee}\right\rangle \geq 0$ for all $\alpha \in \Delta^{P}$. More generally, using (3.4.2), it suffices to show that for any $\alpha \in \Delta^{P}$ and any integer $c \in \mathbb{Z}$ such that $\mathrm{Fil}_{c}^{-\alpha} V_{v} \neq 0$, one has $c \geq 0$. This is trivial by (3.3.1) because $E_{-\alpha}^{(0)}$ is the identity map.

Remark 3.6.5. Proposition 3.6 .4 does not hold in general without the assumption that $P$ is defined over $\mathbb{F}_{q}$, as an example in Subsection 6.2 shows.

\section{The case of $G=\mathrm{SL}_{2, \mathbb{F}_{q}}$}

\subsection{Notation for $\mathrm{SL}_{2}$}

Let $B_{2}$ and $B_{2}^{+}$be the lower-triangular and upper-triangular Borel subgroup of $\mathrm{SL}_{2, k}$. Let $T_{2}$ be the diagonal torus of $\mathrm{SL}_{2, k}$. We put

$$
u_{2}=\left(\begin{array}{ll}
1 & 0 \\
1 & 1
\end{array}\right) \in B_{2}(k)
$$

For $r \in \mathbb{Z}$, let $\chi_{r}$ be the character of $B_{2}$ defined by

$$
\left(\begin{array}{cc}
x & 0 \\
z & x^{-1}
\end{array}\right) \mapsto x^{r}
$$

Let Std: $\mathrm{SL}_{2, k} \rightarrow \mathrm{GL}_{2, k}$ be the standard representation. Restrictions of $\chi_{r}$ and Std to subgroups are denoted by the same notations.

\subsection{Zip datum}

Let $G=\mathrm{SL}_{2, \mathbb{F}_{q}}$ and $\mu: \mathbb{G}_{\mathrm{m}, k} \rightarrow G_{k} ; x \mapsto \operatorname{diag}\left(x, x^{-1}\right)$. Let $Z_{\mu}=(G, P, L, Q, M, \varphi)$ be the associated zip datum. We have $P=B_{2}, Q=B_{2}^{+}$and $L=M=T_{2}$. We take $(B, T)=\left(B_{2}, T_{2}\right)$ as a Borel pair and take a frame as in Lemma 2.2.3. Denote by $\alpha$ the unique element of $\Delta$. In our convention of positivity, $\alpha=\chi_{2}$. Note that $I=\emptyset$ and $\Delta^{P}=\{\alpha\}$. Identify $X^{*}(T)=\mathbb{Z}$ such that $r \in \mathbb{Z}$ corresponds to the character $\chi_{r}$. The zip group $E$ is equal to

$$
\left\{\left(\left(\begin{array}{cc}
a & 0 \\
c & a^{-1}
\end{array}\right),\left(\begin{array}{cc}
a^{q} & b \\
0 & a^{-q}
\end{array}\right)\right) \in B_{2} \times B_{2}^{+}\right\} .
$$

The unique open $E$-orbit $U_{\mu} \subset G_{k}$ is given by

$$
U_{\mu}=\left\{\left(\begin{array}{cc}
x & y \\
z & w
\end{array}\right) \in \mathrm{SL}_{2, k} \mid x \neq 0\right\} .
$$




\subsection{The space $H^{0}\left(G-\mathrm{Zip}^{\mu}, \mathcal{V}(\rho)\right)$}

Let $\rho: B \rightarrow \mathrm{GL}(V)$ be a representation. We write the weight decomposition as $V=\bigoplus_{i \in \mathbb{Z}} V_{i}$ where $T$ acts on $V_{i}$ by the character $\chi_{i}$ for all $i \in \mathbb{Z}$. We have

$$
H^{0}\left(\mathcal{U}_{\mu}, \mathcal{V}(\rho)\right)=V^{L\left(\mathbb{F}_{q}\right)}=\bigoplus_{i \in(q-1) \mathbb{Z}} V_{i}
$$

by Corollary 3.2.3. Because in this case the parabolic $P=B$ is defined over $\mathbb{F}_{q}$, we can apply Corollary 3.4.2 to compute the space of global section

$$
H^{0}\left(G-\operatorname{Zip}^{\mu}, \mathcal{V}(\rho)\right)
$$

Also, because $T$ is split over $\mathbb{F}_{q}$, the map $\wp^{*}$ is given by $v \mapsto-(q-1) v$; hence, $\wp^{*-1}(v)=\frac{-v}{q-1}$. We obtain

$$
H^{0}\left(G-\operatorname{Zip}^{\mu}, \mathcal{V}(\rho)\right)=V^{L\left(\mathbb{F}_{q}\right)} \cap \bigoplus_{i \in \mathbb{Z}} \operatorname{Fil}_{\frac{-\chi_{i}}{q-1}}^{P} V_{i}=\bigoplus_{i \in-(q-1) \mathbb{N}} \operatorname{Fil}_{\frac{-i}{q-1}}^{-\alpha} V_{i},
$$

where we used that $\mathrm{Fil}_{\frac{-i}{q-1}}^{-\alpha} V_{i}=0$ for $i>0$. In particular, $H^{0}\left(G-\mathrm{Zip}^{\mu}, \mathcal{V}(\rho)\right)$ is stable by $T$ and is entirely determined by its weight spaces $\mathrm{Fil}_{\frac{-i}{q-1}}^{-\alpha} V_{i} \subset V_{i}$ for $i \in-(q-1) \mathbb{N}$. Let $(V, \rho) \in \operatorname{Rep}(B)$ and set $n=\operatorname{dim}(V)$. Set $V_{\leq i}=\bigoplus_{j \leq i} V_{j}$ and $V_{\geq i}=\bigoplus_{j \geq i} V_{j}$. Then using Lemma 3.3.1, we have a $B$-stable filtration

$$
\cdots \subset V_{\leq i-1} \subset V_{\leq i} \subset V_{\leq i+1} \subset \cdots
$$

For all $i \in-(q-1) \mathbb{N}$, we have

$$
H^{0}\left(G-\operatorname{Zip}^{\mu}, \mathcal{V}(\rho)\right)_{i}=\left\{\begin{array}{l|l}
f \in V_{i} & \left.\rho\left(u_{2}\right) f \in V_{\geq \frac{(q+1) i}{q-1}}\right\}
\end{array}\right.
$$

by the definition of $\mathrm{Fil}_{\frac{-i}{q-1}}^{-\alpha} V_{i}$.

Lemma 4.3.1. Let $(V, \rho) \in \operatorname{Rep}(B)$ and $m \in \mathbb{Z}$ be the smallest weight of $\rho$. Then one has an inclusion

$$
\bigoplus_{\substack{i \in-(q-1) \mathbb{N},(q+1) i \leq(q-1) m}} V_{i} \subset H^{0}\left(G-\mathrm{Zip}^{\mu}, \mathcal{V}(\rho)\right) .
$$

Proof. Let $f \in V_{i}$ with $i \in-(q-1) \mathbb{N}$ and $(q+1) i \leq(q-1) m$. Then we have $V_{\geq \frac{(q+1) i}{q-1}}=V$, so we have $f \in H^{0}\left(G-\operatorname{Zip}^{\mu}, \mathcal{V}(\rho)\right)_{i}$.

The following example shows that $H^{0}\left(G\right.$-Zip $\left.{ }^{\mu}, \mathcal{V}(\rho)\right)$ is not a sum of weight spaces of $V$ in general.

Example 4.3.2. For $i \in\{1,-1\}$, let $e_{i}$ be a nonzero vector of weight $i$ of Std. Consider $\rho:=\operatorname{Std} \otimes \operatorname{Std}$ with basis $e_{i} \otimes e_{j}$ for $i, j \in\{1,-1\}$. The weights of $\rho$ are $\{2,0,-2\}$, and $\operatorname{dim}\left(V_{2}\right)=\operatorname{dim}\left(V_{-2}\right)=1$, $\operatorname{dim}\left(V_{0}\right)=2$. Then we have

$$
H^{0}\left(G-\operatorname{Zip}^{\mu}, \mathcal{V}(\rho)\right)_{0}=\operatorname{Span}\left(e_{1} \otimes e_{-1}-e_{-1} \otimes e_{1}\right) .
$$

\subsection{Property $(P)$}

Proposition 4.4.1. Let $\rho: B \rightarrow \mathrm{GL}(V)$ be an algebraic representation. Let $m_{1}, \ldots, m_{n}$ be the weights of $V$ ordered so that $m_{1}>m_{2}>\cdots>m_{n}$. The following properties are equivalent:

(i) The subspace $V^{R_{u}(B)}$ is one-dimensional (and hence is equal to $V_{m_{n}}$ ). 
(ii) The intersection of all nonzero B-subrepresentations in $V$ is nonzero.

(iii) For all $1 \leq i \leq n$, we have $\operatorname{dim}\left(V_{m_{i}}\right)=1$ and for any $v \in V_{m_{i}} \backslash\{0\}$, the projection of $\rho\left(u_{2}\right) v$ onto $V_{m_{n}}$ is nonzero.

Proof. We show (i) $\Rightarrow$ (ii). If $W \subset V$ is a nonzero $B$-subrepresentation, then $W^{R_{\mathrm{u}}(B)} \subset V^{R_{\mathrm{u}}(B)}$. Because $W^{R_{\mathrm{u}}(B)} \neq 0$, we have $W^{R_{\mathrm{u}}(B)}=V^{R_{\mathrm{u}}(B)}$ and hence $V^{R_{\mathrm{u}}(B)} \subset W$.

We show (ii) $\Rightarrow$ (iii). We show that for any nonzero $v \in V_{m_{i}}$ the projection of $\rho\left(u_{2}\right) v$ onto $V_{m_{n}}$ is nonzero. For a contradiction, assume that it is zero. Because $B=R_{\mathrm{u}}(B) T$, the $B$-subrepresentation generated by $v$ is generated by $v$ as an $R_{\mathrm{u}}(B)$-representation. Hence, this representation does not have a nontrivial intersection with $V_{m_{n}}$ by Lemma 3.3.1. This contradicts (ii). Hence, the claim follows. We note that $\operatorname{dim} V_{m_{n}}=1$ by (ii). Assume that $\operatorname{dim} V_{m_{i}} \geq 2$ for some $i$. Then there is a nonzero $v \in V_{m_{i}}$ such that the projection to $V_{m_{n}}$ of $\rho\left(u_{2}\right) v$ is zero. This is a contradiction.

We show (iii) $\Rightarrow$ (i). Assume $\operatorname{dim} V^{R_{\mathrm{u}}(B)} \geq 2$. Then $V^{R_{\mathrm{u}}(B)}$ contains $V_{m_{i}}$ for some $i \neq n$. For any nonzero $v \in V_{m_{i}} \subset V^{R_{\mathrm{u}}(B)}$, the projection of $\rho\left(u_{2}\right) v$ onto $V_{m_{n}}$ is zero. This is a contradiction.

We say that $(V, \rho) \in \operatorname{Rep}(B)$ satisfies the property (P) if the equivalent conditions of Proposition 4.4.1 are satisfied.

Example 4.4.2. For $\lambda \in X_{+}^{*}(T)$, the restriction to $B$ of $\operatorname{Ind}_{B}^{G_{k}}(\lambda)$ satisfies the property $(\mathrm{P})$ by the last sentence of Subsection 2.3.

Proposition 4.4.3. Assume that $(V, \rho) \in \operatorname{Rep}(B)$ satisfies the property $(P)$. Then the inclusion (4.3.2) is an equality; that is,

$$
H^{0}\left(G-\operatorname{Zip}^{\mu}, \mathcal{V}(\rho)\right)=\bigoplus_{\substack{i \in-(q-1) \mathbb{N},(q+1) i \leq(q-1) m}} V_{i}
$$

Proof. In this case, the element $\rho\left(u_{2}\right) f$ in equation (4.3.1) has a nonzero projection onto $V_{m}$ by Proposition 4.4.1(iii). Thus, if $f \in H^{0}\left(G \text {-Zip }{ }^{\mu}, \mathcal{V}(\rho)\right)_{i}$, then we must have $m \geq \frac{(q+1) i}{q-1}$. This shows that (4.3.2) is an equality.

\section{Category of automorphic vector bundles on $G-\mathrm{Zip}^{\mu}$}

\subsection{The category $\mathfrak{B} \mathfrak{B}_{P}\left(G-\mathrm{Zip}^{\mu}\right)$}

Recall the functor $\operatorname{Rep}(P) \rightarrow \mathfrak{B} \mathfrak{B}_{P}\left(G\right.$-Zip $\left.{ }^{\mu}\right)$ (Subsection 2.4.2). This functor is not fully faithful even after restricting to the full subcategory $\operatorname{Rep}(L) \subset \operatorname{Rep}(P)$ (see Subsection 2.4.3). Indeed, consider the following example.

Example 5.1.1. Assume that $P$ is defined over $\mathbb{F}_{q}$. Let $1 \in \operatorname{Rep}(L)$ be the trivial $L$-representation and $(V, \rho) \in \operatorname{Rep}(L)$. Then $\operatorname{Hom}_{\operatorname{Rep}(L)}(\mathbf{1}, V)=V^{L}$, whereas we have

$$
\operatorname{Hom}_{\mathfrak{B} B\left(G-\operatorname{Zip}^{\mu}\right)}(\mathcal{V}(\mathbf{1}), \mathcal{V}(\rho))=H^{0}\left(G-Z \operatorname{ip}^{\mu}, \mathcal{V}(\rho)\right)=V^{L\left(\mathbb{F}_{q}\right)} \cap V_{\geq 0}^{\Delta^{P}}
$$

by Corollary 3.4.3.

To overcome the problem, we introduce $L_{\varphi}$-modules with additional structures.

Definition 5.1.2. An $L_{\varphi}$-module with $\Delta^{P}$-monodromy is a pair $((\tau, V), \mathcal{N})$ where $\tau: L_{\varphi} \rightarrow \mathrm{GL}_{k}(V)$ is a finite-dimensional representation of $L_{\varphi}$ with a decomposition $V=\bigoplus_{v \in X^{*}(T)} V_{v}$ as $k$-vector spaces and $\mathcal{N}=\left\{N_{\alpha^{\prime}}^{(j)}\right\}_{\alpha \in \Delta^{P}}, \alpha^{\prime} \in \Xi_{\alpha}, j \in \mathbb{Z}$ is a set of $k$-linear endmorphisms of $V$ such that $N_{\alpha^{\prime}}^{(j)}\left(V_{v}\right) \subset V_{v+j \alpha^{\prime}}$, $N_{\alpha^{\prime}}^{(0)}=\operatorname{Id}$ and $N_{\alpha^{\prime}}^{(j)}=0$ for $j<0$.

Morphisms are given as follows: Let $((\tau, V), \mathcal{N})$ and $\left(\left(\tau^{\prime}, V^{\prime}\right), \mathcal{N}^{\prime}\right)$ be two $L_{\varphi}$-modules with $\Delta^{P}$ monodromy. Then a morphism $((\tau, V), \mathcal{N}) \rightarrow\left(\left(\tau^{\prime}, V^{\prime}\right), \mathcal{N}^{\prime}\right)$ is a $k$-linear map $f: V \rightarrow V^{\prime}$ that satisfies the following: 
(1) $f$ is an $L_{\varphi}$-equivariant morphism.

(2) For $\alpha \in \Delta^{P},[\mathbf{j}] \in \mathbb{Z}^{m_{\alpha}} /\left(\mathbb{Z}^{m_{\alpha}}\right)_{r_{\alpha}}$ and $\chi \in X^{*}(T)$ such that $[\mathbf{j}] \cdot \mathbf{r}_{\alpha}>\delta_{\alpha}(\chi)$, we have

$$
\sum_{\mathbf{j} \in[\mathbf{j}] \mathbf{j}^{\prime} \in \mathbb{Z}^{m_{\alpha}}}(-1)^{\sum_{i=1}^{m_{\alpha}} j_{i}^{\prime}} \operatorname{pr}_{\chi}\left(N_{\alpha_{1}}^{\prime\left(j_{1}^{\prime}\right)} \cdots N_{\alpha_{m_{\alpha}}}^{\prime\left(j_{m_{\alpha}}^{\prime}\right)} f N_{\alpha_{m_{\alpha}}}^{\left(j_{m_{\alpha}}-j_{m_{\alpha}}^{\prime}\right)} \cdots N_{\alpha_{1}}^{\left(j_{1}-j_{1}^{\prime}\right)}\right)=0,
$$

where $\operatorname{pr}_{\chi}$ denotes the projection

$$
\operatorname{pr}_{\chi}: \operatorname{Hom}\left(V, V^{\prime}\right) \simeq \bigoplus_{\nu, v^{\prime} \in X^{*}(T)} \operatorname{Hom}\left(V_{v}, V_{v^{\prime}}^{\prime}\right) \rightarrow \bigoplus_{\nu \in X^{*}(T)} \operatorname{Hom}\left(V_{v}, V_{v+\chi}^{\prime}\right) .
$$

We denote by $L_{\varphi}-\mathrm{MN}_{\Delta^{P}}$ the category of $L_{\varphi}$-modules with $\Delta^{P}$-monodromy.

Remark 5.1.3. The condition (2) in Definition 5.1.2 means that $f$ is compatible with $\mathcal{N}$ and $\mathcal{N}^{\prime}$ in some sense. Assume that $P$ is defined over $\mathbb{F}_{q}$. Then the condition (2) in Definition 5.1.2 is simplified as follows: For $\alpha \in \Delta^{P}, \chi \in X^{*}(T)$ and $j \in \mathbb{N}$ such that $j r_{\alpha, 1}>\delta_{\alpha}(\chi)$, we have

$$
\operatorname{pr}_{\chi}\left(\sum_{0 \leq j^{\prime} \leq j}(-1)^{j^{\prime}} N_{-\alpha}^{\left(j^{\prime}\right)} f N_{-\alpha}^{\left(j-j^{\prime}\right)}\right)=0 .
$$

The morphism $N_{-\alpha}^{(j)}$ is an analogue of $N^{j} / j$ ! for a monodromy operator $N$ in characteristic zero. In this sense,

$$
f \mapsto \sum_{0 \leq j^{\prime} \leq j}(-1)^{j^{\prime}} N_{-\alpha}^{\left(j^{\prime}\right)} f N_{-\alpha}^{\left(j-j^{\prime}\right)}
$$

is an analogue of $j$ th iterate of

$$
f \mapsto f N-N^{\prime} f
$$

divided by $j$ ! for monodromy operators $N$ and $N^{\prime}$ in characteristic zero.

We have the functor

$$
F_{\mathrm{MN}}: \operatorname{Rep}(P) \rightarrow L_{\varphi^{-}} \mathrm{MN}_{\Delta^{P}} ;(V, \rho) \mapsto\left(\left(V,\left.\rho\right|_{L_{\varphi}}\right),\left\{E_{\alpha^{\prime}}^{(j)}\right\}_{\alpha \in \Delta^{P}, \alpha^{\prime} \in \Xi_{\alpha}, j \in \mathbb{Z}}\right)
$$

where we equip $V$ with the natural $T$-weight decomposition $V=\bigoplus_{v} V_{v}$.

Definition 5.1.4. An $L_{\varphi}$-module with $\Delta^{P}$-monodromy is called admissible if it is in the essential image of $F_{\mathrm{MN}}$. We denote by $L_{\varphi}$-MN $\Delta^{P}$ adm category of admissible $L_{\varphi}$-modules with $\Delta^{P}$-monodromy.

Theorem 5.1.5. The functor $\mathcal{V}: \operatorname{Rep}(P) \rightarrow \mathfrak{B B}\left(G\right.$-Zip $\left.{ }^{\mu}\right)$ factors through the functor $F_{\mathrm{MN}}: \operatorname{Rep}(P) \rightarrow$ $L_{\varphi}-\mathrm{MN}_{\Delta^{P}}^{\mathrm{adm}}$ and induces an equivalence of categories

$$
L_{\varphi}-\mathrm{MN}_{\Delta^{P}}^{\mathrm{adm}} \longrightarrow \mathfrak{B} \mathfrak{B}_{P}\left(G-\mathrm{Zip}^{\mu}\right)
$$

Proof. For two $P$-representations $(V, \rho)$ and $\left(V^{\prime}, \rho^{\prime}\right)$, one has

$$
\begin{aligned}
\operatorname{Hom}_{\mathfrak{B} B\left(G-\mathrm{Zip}^{\mu}\right)}\left(\mathcal{V}(\rho), \mathcal{V}\left(\rho^{\prime}\right)\right) & =\operatorname{Hom}_{\mathfrak{B} B\left(G-Z \mathrm{Zip}^{\mu}\right)}\left(\mathcal{V}(\mathbf{1}), \mathcal{V}(\rho)^{\vee} \otimes \mathcal{V}\left(\rho^{\prime}\right)\right) \\
& =\operatorname{Hom}_{\mathfrak{B} B\left(G-Z i^{\mu}\right)}\left(\mathcal{V}(\mathbf{1}), \mathcal{V}\left(\rho^{\vee} \otimes \rho^{\prime}\right)\right) \\
& =H^{0}\left(G-Z \mathrm{Zip}^{\mu}, \mathcal{V}\left(\rho^{\vee} \otimes \rho^{\prime}\right)\right) \\
& =\left(V^{\vee} \otimes V^{\prime}\right)^{L_{\varphi}} \cap \bigcap_{\alpha \in \Delta^{P}[v] \in X^{*}(T) / \Lambda_{\Xi_{\alpha}, \mathbf{r}_{\alpha}}} \bigoplus_{\delta_{\alpha}}^{\Xi_{\alpha}, \mathbf{a}_{\alpha}, \mathbf{r}_{\alpha}}\left(V^{\vee} \otimes V^{\prime}\right)_{[v]},
\end{aligned}
$$


where we used Theorem 3.4.1 in the last line. We can see from the definition that this space coincides with the space of homomorphisms $F_{\mathrm{MN}}(V, \rho) \rightarrow F_{\mathrm{MN}}\left(V^{\prime}, \rho^{\prime}\right)$ using that the action of $u_{\alpha^{\prime}}(x)$ on $V^{\vee} \otimes V^{\prime}$ is given by $f \mapsto \rho^{\prime}\left(u_{\alpha^{\prime}}(x)\right) \circ f \circ \rho\left(u_{\alpha^{\prime}}(-x)\right)$ for $\alpha^{\prime} \in \Xi_{\alpha}$.

Let $S_{K}$ denote the good reduction special fibre of a Hodge-type Shimura variety, with the same notations and assumptions as in Subsection 2.5. Recall that there is a functor $\mathcal{V}: \operatorname{Rep}(P) \rightarrow \mathfrak{B} \mathfrak{B}\left(S_{K}\right)$ (see (2.5.1)), which induces functors

$$
\operatorname{Rep}(P) \stackrel{\mathcal{V}}{\longrightarrow} \mathfrak{B} \mathfrak{B}_{P}\left(G-\mathrm{Zip}^{\mu}\right) \stackrel{\zeta^{*}}{\longrightarrow} \mathfrak{B} \mathfrak{B}_{P}\left(S_{K}\right),
$$

where $\mathfrak{B} \mathfrak{B}_{P}\left(S_{K}\right)$ also denotes the essential image of $\operatorname{Rep}(P)$ in $\mathfrak{B} \mathfrak{B}\left(S_{K}\right)$. We obtain the following corollary in the context of Shimura varieties.

Corollary 5.1.6. The functor $\mathcal{V}: \operatorname{Rep}(P) \rightarrow \mathfrak{B B}_{P}\left(S_{K}\right)$ factors as

$$
\operatorname{Rep}(P) \stackrel{F_{\mathrm{MN}}}{\longrightarrow} L_{\varphi}-\mathrm{MN}_{\Delta^{P}}^{\mathrm{adm}} \stackrel{\zeta^{*}}{\longrightarrow} \mathfrak{B} \mathfrak{B}_{P}\left(S_{K}\right) .
$$

\subsection{The category $\mathfrak{B B}_{L}\left(G-\mathrm{Zip}^{\mu}\right)$}

We assume that $P$ is defined over $\mathbb{F}_{q}$. Hence, in what follows, we have $L_{\varphi}=L\left(\mathbb{F}_{q}\right)$.

Definition 5.2.1. Let $\mathfrak{B B}_{L}\left(G\right.$-Zip $\left.{ }^{\mu}\right)$ denote the full subcategory of $\mathfrak{B} \mathfrak{B}\left(G-Z i p^{\mu}\right)$, which is equal to the essential image of the functor $\operatorname{Rep}(L) \rightarrow \mathfrak{B B}\left(G\right.$-Zip $\left.{ }^{\mu}\right)$. We call it the category of $L$-vector bundles on $G$-Zip ${ }^{\mu}$.

For example, the automorphic vector bundles $(\mathcal{V}(\lambda))_{\lambda \in X^{*}(T)}$ (see Subsection 2.4.3) lie in the subcategory of $L$-vector bundles on $G$-Zip ${ }^{\mu}$.

Definition 5.2.2. A $\Delta^{P}$-filtered $L_{\varphi}$-module is a pair $((\tau, V), \mathcal{F})$ where $\tau: L_{\varphi} \rightarrow \mathrm{GL}_{k}(V)$ is a finitedimensional representation of $L_{\varphi}$ and $\mathcal{F}=\left\{V_{\geq \bullet}^{\alpha}\right\}_{\alpha \in \Delta^{P}}$ is a set of filtrations on $V$. Here, $V_{\geq \bullet}^{\alpha}$ denotes a

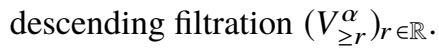

Morphisms are given as follows. Let $((\tau, V), \mathcal{F})$ and $\left(\left(\tau^{\prime}, V^{\prime}\right), \mathcal{F}^{\prime}\right)$ be two $\Delta^{P}$-filtered $L_{\varphi}$-modules. Then a morphism $((\tau, V), \mathcal{F}) \rightarrow\left(\left(\tau^{\prime}, V^{\prime}\right), \mathcal{F}^{\prime}\right)$ is a $k$-linear map $f: V \rightarrow V^{\prime}$ that satisfies the following:

(1) $f$ is an $L_{\varphi}$-equivariant morphism.

(2) For each $\alpha \in \Delta^{P}$, the map $f$ is compatible with the filtrations $V_{\geq \bullet}^{\alpha}$ and $V_{\geq \bullet}^{\prime \alpha}$ in the sense that $f\left(V_{\geq r}^{\alpha}\right) \subset V_{\geq r}^{\prime \alpha}$ for any $r \in \mathbb{R}$.

We denote by $L_{\varphi}-\mathrm{MF}_{\Delta^{P}}^{\mathrm{adm}}$ the category of $\Delta^{P}$-filtered $L_{\varphi}$-modules.

Let $((\tau, V), \mathcal{N}) \in L_{\varphi}-\mathrm{MN}_{\Delta^{P}}$. For $\alpha \in \Delta^{P}$, define the $\alpha$-filtration $\left(V_{\geq \bullet}^{\alpha}\right)$ of $V$ as follows: Let $V=$ $\bigoplus_{v} V_{v}$ be the weight decomposition of $V$. For all $r \in \mathbb{R}$, let $V_{\geq r}^{\alpha}$ be the direct sum of $V_{v}$ for all $v$ satisfying $\left\langle v, \delta_{\alpha}\right\rangle \geq r$. We call $V_{\geq \bullet}^{\alpha}$ the $\alpha$-filtration of $V$. Thus, we have a functor $L_{\varphi^{-}}-\mathrm{MN}_{\Delta^{P}} \rightarrow L_{\varphi^{-}}-\mathrm{MF}_{\Delta^{P}}$. Taking composition, we obtain

$$
F_{\mathrm{MF}}: \operatorname{Rep}(L) \rightarrow \operatorname{Rep}(P) \stackrel{F_{\mathrm{MN}}}{\longrightarrow} L_{\varphi^{-}}-\mathrm{MN}_{\Delta^{P}} \rightarrow L_{\varphi^{-}}-\mathrm{MF}_{\Delta^{P}}
$$

Definition 5.2.3. A $\Delta^{P}$-filtered $L_{\varphi}$-module is called admissible if it is in the essential image of $F_{\text {MF. }}$ We denote by $L_{\varphi}-\mathrm{MF}_{\Delta^{P}}^{\text {adm }}$ the category of admissible $\Delta^{P}$-filtered $L_{\varphi}$-modules.

Theorem 5.2.4. The functor $V: \operatorname{Rep}(L) \rightarrow \mathfrak{B B}\left(G\right.$-Zip $\left.{ }^{\mu}\right)$ factors through the functor $F_{M F}: \operatorname{Rep}(L) \rightarrow$ $L_{\varphi}-M F_{\Delta^{P}}^{\mathrm{adm}}$ and induces an equivalence of categories

$$
L_{\varphi}-M F_{\Delta^{P}}^{\mathrm{adm}} \longrightarrow \mathfrak{B} \mathfrak{B}_{L}\left(G-\mathrm{Zip}^{\mu}\right)
$$


Proof. By Theorem 5.1.5, it suffices to show

$$
\operatorname{Hom}_{L_{\varphi}-\mathrm{MN}_{\Delta} P}\left(F_{\mathrm{MN}}(\rho), F_{\mathrm{MN}}\left(\rho^{\prime}\right)\right)=\operatorname{Hom}_{L_{\varphi}-\mathrm{MF}_{\Delta} P}\left(F_{\mathrm{MF}}(\rho), F_{\mathrm{MF}}\left(\rho^{\prime}\right)\right)
$$

for $(V, \rho),\left(V^{\prime}, \rho^{\prime}\right) \in \operatorname{Rep}(L)$. This follows from Remark 5.1.3 and the definitions of morphisms in $L_{\varphi}-\mathrm{MN}_{\Delta^{P}}$ and $L_{\varphi}-\mathrm{MF}_{\Delta^{P}}$.

\section{Examples}

\subsection{The algebras $R_{I}$ and $R_{\Delta}$}

Fix a connected reductive group $G$ over $\mathbb{F}_{q}$, a cocharacter $\mu: \mathbb{G}_{\mathrm{m}, k} \rightarrow G_{k}$ and a frame $(B, T, z)$ for $z_{\mu}$ (Subsection 2.2.3). For $\lambda \in X_{+}^{*}(T)$, denote by $V_{\Delta}(\lambda)$ the $G$-representation $\operatorname{Ind}_{B}^{G}(\lambda)$. We add a subscript $\Delta$ to avoid confusion with $V_{I}(\lambda)=\operatorname{Ind}_{B_{L}}^{L}(\lambda)$ for $\lambda \in X_{+, I}^{*}(T)$ (see $\S 2.4$.3). Let $\mathcal{V}_{\Delta}(\lambda)$ be the vector bundle on $G$-Zip ${ }^{\mu}$ attached to $V_{\Delta}(\lambda)$. We put

$$
R_{I}=\bigoplus_{\lambda \in X_{+, I}^{*}(T)} H^{0}\left(G-\mathrm{Zip}^{\mu}, \nu_{I}(\lambda)\right) \quad \text { and } \quad R_{\Delta}=\bigoplus_{\lambda \in X_{+}^{*}(T)} H^{0}\left(G-\mathrm{Zip}^{\mu}, \nu_{\Delta}(\lambda)\right)
$$

By (2.3.3), the $k$-vector spaces $R_{I}$ and $R_{\Delta}$ have a natural structure of $k$-algebra. They capture information about all $\mathcal{V}_{I}(\lambda)$ and $\mathcal{V}_{\Delta}(\lambda)$ at once.

Remark 6.1.1. In general, we do not know whether $R_{I}$ and $R_{\Delta}$ are finite-type algebras, but we conjecture that this is the case. The algebra $R_{I}$ was studied in [Kos19]. In the case of $G=\operatorname{Sp}(4)$ with a cocharacter $\mu$ whose centraliser Levi subgroup is isomorphic to $\mathrm{GL}_{2}$, we showed that $R_{I}$ is a polynomial algebra in three indeterminates [Kos19, Theorem 5.4.1].

In this first example, we examine $R_{\Delta}$ in the case of $G=\mathrm{SL}_{2, \mathbb{F}_{q}}$ with the zip datum explained in Subsection 4.2. In this case, the algebra $R_{I}$ is very simple: It is a polynomial algebra in one indeterminate, generated by the classical Hasse invariant. Let $n \in \mathbb{N}$. The representation $V_{\Delta}\left(\chi_{n}\right)$ identifies with $\operatorname{Sym}^{n}(\mathrm{Std})$. The weights of $V_{\Delta}\left(\chi_{n}\right)$ are $\{-n+2 i \mid 0 \leq i \leq n\}$. By Example 4.4.2 and Proposition 4.4.3, we have

$$
H^{0}\left(G-\operatorname{Zip}^{\mu}, \mathcal{V}_{\Delta}\left(\chi_{n}\right)\right)=\bigoplus_{\substack{i \in-(q-1) \mathbb{N},(q+1) i \leq-(q-1) n}} V_{\Delta}\left(\chi_{n}\right)_{i}
$$

for all $n \geq 0$. Let $x, y$ be indeterminates. Let $\mathrm{SL}_{2}$ act on $k[x, y]$ by

$$
\left(\begin{array}{ll}
a & b \\
c & d
\end{array}\right) \cdot P=P(a x+c y, b x+d y)
$$

Then $V_{\Delta}\left(\chi_{n}\right)=\operatorname{Sym}^{n}(\operatorname{Std})$ is the subrepresentation of $k[x, y]$ spanned by homogeneous polynomials in $x, y$ of degree $n$. The highest weight vector is $x^{n}$. By (6.1.1), we have

$$
H^{0}\left(G-\operatorname{Zip}^{\mu}, \mathcal{V}_{\Delta}\left(\chi_{n}\right)\right)=\operatorname{Span}_{k}\left(x^{j} y^{n-j}|j \geq 0, q-1| n-2 j,(q+1) j \leq n\right) .
$$

Similarly, $R_{\Delta}$ is the subalgebra of $k[x, y]$ generated by $x^{j} y^{n-j}$ for all $0 \leq j \leq n$ with $q-1 \mid n-2 j$ and $(q+1) j \leq n$.

Proposition 6.1.2. The algebra $R_{\Delta}$ is generated by $y^{q-1}$ and $x y^{q}$. In particular, it is a polynomial algebra in two indeterminates.

Proof. It is clear that $y^{q-1}$ and $x y^{q}$ are elements of $R_{\Delta}$. Let $n \geq 0$ and $0 \leq j \leq n$ such that $x^{j} y^{n-j} \in R_{\Delta}$. We can write $x^{j} y^{n-j}=\left(x y^{q}\right)^{j} y^{n-(q+1) j}$. Note that $n \geq(q+1) j$ and $q-1$ divides $n-(q+1) j=$ $n-2 j-(q-1) j$. It follows that $x^{j} y^{n-j}$ lies in the subalgebra of $k[x, y]$ generated by $y^{q-1}$ and $x y^{q}$. 
We give an interpretation of these sections. In the case of $G=\mathrm{SL}_{2, \mathbb{F}_{q}}$, recall that for an $\mathbb{F}_{q}$-scheme $S$, the groupoid $G$-Zip ${ }^{\mu}(S)$ consists of tuples $\underline{\mathcal{H}}=(\mathcal{H}, \omega, F, V)$ where

(1) $\mathcal{H}$ is a locally free $\mathcal{O}_{S}$-module of rank 2 with a trivialisation $\operatorname{det}(\mathcal{H}) \simeq \mathcal{O}_{S}$,

(2) $\omega \subset \mathcal{H}$ is a locally free $\mathcal{O}_{S}$-submodule of rank 1 such that $\mathcal{H} / \omega$ is locally free and

(3) $F: \mathcal{H}^{(q)} \rightarrow \mathcal{H}$ and $V: \mathcal{H} \rightarrow \mathcal{H}^{(q)}$ are $\mathcal{O}_{S}$-linear maps satisfying the conditions $\operatorname{Ker}(F)=\operatorname{Im}(V)=$ $\omega^{(q)}$ and $\operatorname{Ker}(V)=\operatorname{Im}(F)$.

Consider the flag space $\mathcal{F}_{G}$ over $G$-Zip ${ }^{\mu}$ parametrising pairs $(\underline{\mathcal{H}}, \mathcal{L})$ with $\mathcal{L} \subset \mathcal{H}$ a locally free $\mathcal{O}_{S^{-}}$ submodule of rank 1 such that $\mathcal{H} / \mathcal{L}$ is locally free. The natural projection map $\pi_{G}: \mathcal{F}_{G} \rightarrow G$-Zip ${ }^{\mu}$ is a $\mathbb{P}^{1}$-fibration. For $n \in \mathbb{Z}$, the push-forward $\pi_{G, *}\left(\mathcal{L}^{-n}\right)$ coincides with the vector bundle $\mathcal{V}_{\Delta}\left(\chi_{n}\right)$. Consider the map

$$
\mathcal{L} \subset \mathcal{H} \stackrel{V}{\rightarrow} \mathcal{H}^{(q)} \rightarrow(\mathcal{H} / \mathcal{L})^{(q)} \simeq \mathcal{L}^{-q},
$$

where we used that $\mathcal{H} / \mathcal{L} \simeq \mathcal{L}^{-1}$ by the trivialisation $\operatorname{det}(\mathcal{H}) \simeq \mathcal{O}_{S}$. We obtain a section of $\mathcal{L}^{-(q+1)}$. It corresponds to the element $x y^{q}$ in Proposition 6.1.2. On the other hand, the classical Hasse invariant $H a \in H^{0}\left(S, \omega^{q-1}\right)$ is given by the map $V: \omega \rightarrow \omega^{(q)} \simeq \omega^{q}$. By sending Ha under the morphism

$$
\omega \subset \mathcal{H} \rightarrow \mathcal{H} / \mathcal{L} \simeq \mathcal{L}^{-1}
$$

we obtain a section of $\mathcal{L}^{-(q-1)}$. This section corresponds to $y^{q-1}$ in Proposition 6.1.2.

\subsection{Example on L-semisimplification}

We give an example that shows that Proposition 3.6.4 does not hold in general without the assumption that $P$ is defined over $\mathbb{F}_{q}$. Let $G=\operatorname{Res}_{\mathbb{F}_{q^{2}} / \mathbb{F}_{q}} \mathrm{SL}_{2, \mathbb{F}_{q^{2}}}$ and

$$
\mu: \mathbb{G}_{\mathrm{m}, k} \rightarrow G_{k} \simeq \mathrm{SL}_{2, k} \times \mathrm{SL}_{2, k} ; z \mapsto\left(\left(\begin{array}{cc}
z & 0 \\
0 & z^{-1}
\end{array}\right),\left(\begin{array}{ll}
1 & 0 \\
0 & 1
\end{array}\right)\right) .
$$

Let $z_{\mu}=(G, P, L, Q, M, \varphi)$ be the associated zip datum. We have $P=B_{2} \times \mathrm{SL}_{2, k}, L=T_{2} \times \mathrm{SL}_{2, k}$, $Q=\mathrm{SL}_{2, k} \times B_{2}^{+}$and $M=\mathrm{SL}_{2, k} \times T_{2}$. We take $(B, T)=\left(B_{2} \times B_{2}, T_{2} \times T_{2}\right)$ as a Borel pair and take a frame as in Lemma 2.2.3. Then $\Delta^{P}$ consists of one root $\alpha=\chi_{2} \otimes \chi_{0}$. We have

$$
L_{\varphi}=\left\{\left(\left(\begin{array}{cc}
x & 0 \\
0 & x^{-1}
\end{array}\right),\left(\begin{array}{cc}
x^{q} & y \\
0 & x^{-q}
\end{array}\right)\right) \in L \mid x \in \mathbb{F}_{q^{2}}^{\times}, y^{q}=0\right\} .
$$

We have

$$
\begin{aligned}
& \delta_{\alpha}=\frac{-\alpha^{\vee}-q \sigma\left(\alpha^{\vee}\right)}{q^{2}-1}, \quad \mathbf{r}_{\alpha}=\left(\frac{q^{2}+1}{q^{2}-1}, \frac{-\left(q^{2}+1\right)}{q\left(q^{2}-1\right)}\right), \\
& \left(\mathbb{Z}^{2}\right)_{\mathbf{r}_{\alpha}}=\left\{\left(n_{1}, n_{2}\right) \in \mathbb{Z}^{2} \mid q n_{1}=n_{2}\right\} .
\end{aligned}
$$

We define $\rho: P \rightarrow \mathrm{GL}(V)$ by

$$
\left(\operatorname{Sym}^{q^{2}-1}(\operatorname{Std}) \otimes \chi_{q^{2}-1}\right) \otimes \operatorname{Sym}^{q^{2}-1}\left(\operatorname{Std}^{(q)}\right) .
$$

We write $\left(V^{\prime}, \rho^{\prime}\right)$ for $\left(V^{L \text {-ss }}, \rho^{L \text {-ss }}\right)$. Then we have $V^{L_{\varphi}}=V$ and $V^{\prime L_{\varphi}}=V^{\prime}$. We put $v=\chi_{0} \chi_{-q\left(q^{2}-3\right)}$. We have

$$
V_{[v]}=V_{v} \oplus V_{v+\alpha-q \sigma(\alpha)} .
$$


We parametrise elements $[\mathbf{j}] \in \mathbb{Z}^{2} /\left(\mathbb{Z}^{2}\right)_{\mathbf{r}_{\alpha}}$ by classes $[(0, j)]$ with $j \in \mathbb{Z}$. Using this notation, we have

$$
\operatorname{Fil}_{\delta_{\alpha}}^{\Xi_{\alpha}, \mathbf{a}_{\alpha}, \mathbf{r}_{\alpha}} V_{[v]}=\bigcap_{j \in \mathbb{Z}} \bigcap_{\substack{\chi \in[v+j \sigma(\alpha)] \\ j r_{\alpha, 2}>\delta_{\alpha}(\chi)}} \operatorname{Ker}\left(\sum_{j_{1} \in \mathbb{Z}} \operatorname{pr}_{\chi} \circ E_{-\alpha}^{\left(j_{1}\right)} \circ E_{\sigma(\alpha)}^{\left(j+q j_{1}\right)}: V_{[v]} \rightarrow V_{\chi}\right)
$$

because $(-1)^{j_{1}}(-1)^{j+q j_{1}}=(-1)^{j} \in k$. We have $V_{\chi} \neq 0$ if and only if $\chi=v+i_{1} \alpha+q i_{2} \sigma(\alpha)$ for $0 \leq i_{1} \leq q^{2}-1$ and $-1 \leq i_{2} \leq q^{2}-2$. For $\chi=v+i_{1} \alpha+q i_{2} \sigma(\alpha)$, the conditions $\chi \in[v+j \sigma(\alpha)]$ and $j r_{\alpha, 2}>\delta_{\alpha}(\chi)$ hold if and only if $j=q\left(i_{1}+i_{2}\right)$ and $i_{2}-i_{1}>q^{2}-2-2 /\left(q^{2}-1\right)$. Hence,

$$
\chi \in[v+j \sigma(\alpha)], j r_{\alpha, 2}>\delta_{\alpha}(\chi), V_{\chi} \neq 0 \Longleftrightarrow \chi=v+q\left(q^{2}-2\right) \sigma(\alpha), j=q\left(q^{2}-2\right) .
$$

We put $\chi_{0}=v+q\left(q^{2}-2\right) \sigma(\alpha)$ and $j_{0}=q\left(q^{2}-2\right)$. Then we have

$$
\begin{aligned}
\operatorname{Fil}_{\delta_{\alpha}}^{\Xi_{\alpha}, \mathbf{a}_{\alpha}, \mathbf{r}_{\alpha}} V_{[v]} & =\operatorname{Ker}\left(\operatorname{pr}_{\chi_{0}} \circ\left(E_{\sigma(\alpha)}^{\left(j_{0}\right)}+E_{-\alpha}^{(1)} \circ E_{\sigma(\alpha)}^{\left(j_{0}+q\right)}\right): V_{[v]} \rightarrow V_{\chi_{0}}\right) \\
& =\left\{\left(v_{1}, v_{2}\right) \in V_{v} \oplus V_{v+\alpha-q \sigma(\alpha)} \mid E_{\sigma(\alpha)}^{\left(j_{0}\right)}\left(v_{1}\right)+\left(E_{-\alpha}^{(1)} \circ E_{\sigma(\alpha)}^{\left(j_{0}+q\right)}\right)\left(v_{2}\right)=0\right\} .
\end{aligned}
$$

We note that

$$
E_{\sigma(\alpha)}^{\left(j_{0}\right)}: V_{v} \rightarrow V_{\chi_{0}}, \quad E_{-\alpha}^{(1)} \circ E_{\sigma(\alpha)}^{\left(j_{0}+q\right)}: V_{\nu+\alpha-q \sigma(\alpha)} \rightarrow V_{\chi_{0}}
$$

are isomorphisms. In the same way, we have

$$
\operatorname{Fil}_{\delta_{\alpha}}^{\Xi_{\alpha}, \mathbf{a}_{\alpha}, \mathbf{r}_{\alpha}} V_{[v]}^{\prime}=\operatorname{Ker}\left(\operatorname{pr}_{\chi_{0}} \circ E_{\sigma(\alpha)}^{\left(j_{0}\right)}: V_{[v]}^{\prime} \rightarrow V_{\chi 0}^{\prime}\right)=V_{v+\alpha-q \sigma(\alpha)}^{\prime}
$$

using $E_{-\alpha}^{(1)}=0$ for $\left(V^{\prime}, \rho^{\prime}\right)$. Hence, $\mathrm{Fil}_{\delta_{\alpha}}^{\Xi_{\alpha}, \mathbf{a}_{\alpha}, \mathbf{r}_{\alpha}} V_{[v]} \not \subset \mathrm{Fil}_{\delta_{\alpha}}^{\Xi_{\alpha}, \mathbf{a}_{\alpha}, \mathbf{r}_{\alpha}} V_{[v]}^{\prime}$. Therefore, we have $H^{0}\left(G\right.$-Zip $\left.^{\mu}, \mathcal{V}(\rho)\right) \not \subset H^{0}\left(G-\right.$ Zip $\left.^{\mu}, \mathcal{V}\left(\rho^{\prime}\right)\right)$.

\subsection{The case of the unitary group $U(2,1)$ with $p$ inert}

In this subsection, we examine an example that arises in the study of Picard surfaces. These are Shimura varieties of PEL type (in particular, of Hodge type) attached to unitary groups $\mathbf{G}$ over $\mathbb{Q}$ with respect to some totally imaginary quadratic extension $\mathbf{E} / \mathbb{Q}$. We impose that $\mathbf{G}_{\mathbb{R}} \simeq \mathrm{GU}(2,1)$. We choose a rational prime $p$ that is inert in $\mathbf{E}$ and consider the attached zip datum $(G, P, Q, L, M, \varphi)$. Because $p$ is inert, the parabolic $P$ is not defined over $\mathbb{F}_{p}$. We study the space $H^{0}\left(G\right.$-Zip $\left.{ }^{\mu}, \mathcal{V}_{I}(\lambda)\right)$. To simplify, we will work with a unitary group $U$, instead of a group of unitary similitudes GU. The case of GU is very similar.

Let $(V, \psi)$ be a 3-dimensional vector space over $\mathbb{F}_{q^{2}}$ endowed with a nondegenerate Hermitian form $\psi: V \times V \rightarrow \mathbb{F}_{q^{2}}$ (in the context of Shimura varieties, take $q=p$ ). Write $\operatorname{Gal}\left(\mathbb{F}_{q^{2}} / \mathbb{F}_{q}\right)=\{\mathrm{Id}, \sigma\}$. We take a basis $\mathcal{B}=\left(v_{1}, v_{2}, v_{3}\right)$ of $V$ where $\psi$ is given by the matrix

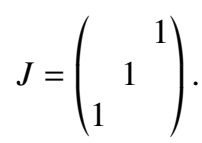

We define a reductive group $G$ by

$$
G(R)=\left\{f \in \mathrm{GL}_{\mathbb{F}_{q^{2}}}\left(V \otimes_{\mathbb{F}_{q}} R\right) \mid \psi_{R}(f(x), f(y))=\psi_{R}(x, y), \forall x, y \in V \otimes_{\mathbb{F}_{q}} R\right\}
$$

for any $\mathbb{F}_{q^{-}}$-alegebra $R$. One has an identification $G_{\mathbb{F}_{q^{2}}} \simeq \mathrm{GL}(V)$, given as follows: For any $\mathbb{F}_{q^{2}}$-algebra

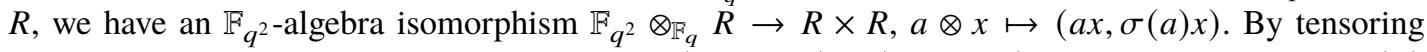
with $V$, we obtain an isomorphism $V \otimes_{\mathbb{F}_{q}} R \rightarrow\left(V \otimes_{\mathbb{F}_{q^{2}}} R\right) \oplus\left(V \otimes_{\mathbb{F}_{q^{2}}} R\right)$. Then any element of $G(R)$ 
stabilises this decomposition and is entirely determined by its restriction to the first summand. This yields an isomorphism as claimed. Using the basis $\mathcal{B}$, we identify $G_{\mathbb{F}_{q^{2}}}$ with $\mathrm{GL}_{3, \mathbb{F}_{q^{2}}}$. The action of $\sigma$ on the set $\mathrm{GL}_{3}(k)$ is given as follows: $\sigma \cdot A=J \sigma\left({ }^{t} A\right)^{-1} J$. Let $T$ denote the maximal diagonal torus and $B$ the lower-triangular Borel subgroup of $G_{k}$. Note that by our choice of the basis $\mathcal{B}$, the groups $B$ and $T$ are defined over $\mathbb{F}_{q}$. Identify $X^{*}(T)=\mathbb{Z}^{3}$ such that $\left(k_{1}, k_{2}, k_{3}\right) \in \mathbb{Z}^{3}$ corresponds to the character $\operatorname{diag}\left(x_{1}, x_{2}, x_{3}\right) \mapsto \prod_{i=1}^{3} x_{i}^{k_{i}}$. The simple roots are $\Delta=\left\{e_{1}-e_{2}, e_{2}-e_{3}\right\}$, where $\left(e_{1}, e_{2}, e_{3}\right)$ is the canonical basis of $\mathbb{Z}^{3}$.

Define a cocharacter $\mu: \mathbb{G}_{\mathrm{m}, k} \rightarrow G_{k}$ such that $\mu$ is given by $x \mapsto \operatorname{diag}(x, x, 1)$ via the identification $G_{k} \simeq \mathrm{GL}_{3, k}$. Let $z_{\mu}=(G, P, L, Q, M, \varphi)$ be the associated zip datum. Note that $P$ is not defined over $\mathbb{F}_{q}$. One has $I=\left\{e_{1}-e_{2}\right\}$ and $\Delta^{P}=\{\alpha\}$ with $\alpha=e_{2}-e_{3}$.

Lemma 6.3.1. Let $H$ be the function on $G_{k}$ defined by

$$
H\left(\left(x_{i, j}\right)_{1 \leq i, j \leq 3}\right)=x_{1,1}^{q} \Delta_{1}-x_{2,1}^{q} \Delta_{2} \quad \text { with }\left\{\begin{array}{l}
\Delta_{1}=x_{1,1} x_{2,2}-x_{1,2} x_{2,1}, \\
\Delta_{2}=x_{1,1} x_{2,3}-x_{2,1} x_{1,3} .
\end{array}\right.
$$

The $\mu$-ordinary stratum $U_{\mu} \subset G_{k}$ is equal to the complement of the vanishing locus of $H$.

Proof. In this case, there is a unique $E$-orbit of codimension 1 by the first part of Theorem 2.2.4. Furthermore, this $E$-orbit is dense in $G_{k} \backslash U_{\mu}$ by the closure relation. Hence, it suffices to show that $H$ does not vanish on $U_{\mu}$. The group $E$ consists of pairs $(x, y) \in P \times Q$ with

$$
x=\left(\begin{array}{lll}
a & b & 0 \\
c & d & 0 \\
e & f & g
\end{array}\right) \quad \text { and } \quad y=\left(\begin{array}{ccc}
g^{q} & h & i \\
0 & d^{q} & b^{q} \\
0 & c^{q} & a^{q}
\end{array}\right)^{-1}
$$

Because $1 \in U_{\mu}$, the open $U_{\mu}$ consists of elements of the form $x y^{-1}$. We find

$$
H\left(x y^{-1}\right)=\left(a g^{q}\right)^{q} g^{q} d^{q}(a d-b c)-\left(c g^{q}\right)^{q} g^{q} b^{q}(a d-b c)=g^{q^{2}+q}(a d-b c)^{q+1} .
$$

This expression is nonzero, so the result is proved.

We have

$$
L_{\varphi}=\left\{\left(\begin{array}{ccc}
a & b & \\
& d & \\
& & a^{-q}
\end{array}\right) \in L \mid a, d \in \mathbb{F}_{q^{2}}^{\times}, d^{q+1}=1, b^{q}=0\right\} .
$$

The endomorphism $\wp_{*}: X_{*}(T)_{\mathbb{R}} \rightarrow X_{*}(T)_{\mathbb{R}}$ is given by the matrix

$$
\wp_{*}=\left(\begin{array}{ccc}
1 & & q \\
& 1+q & \\
q & & 1
\end{array}\right) .
$$

Hence, it follows that $\delta_{\alpha}=\wp_{*}^{-1}\left(\alpha^{\vee}\right)=\frac{1}{q^{2}-1}(-q, q-1,1)$. We have $m_{\alpha}=2, \mathbf{a}_{\alpha}=(-1,-1), \Xi_{\alpha}=$ $(-\alpha, \sigma(\alpha))$ and

$$
\mathbf{r}_{\alpha}=\left(\frac{q^{2}-q+1}{q^{2}-1}, \frac{-q^{2}+q-1}{q\left(q^{2}-1\right)}\right), \quad\left(\mathbb{Z}^{2}\right)_{\mathbf{r}_{\alpha}}=\left\{\left(n_{1}, n_{2}\right) \in \mathbb{Z}^{2} \mid q n_{1}=n_{2}\right\} .
$$

The group $\Lambda_{\Xi_{\alpha}, \mathbf{r}_{\alpha}}$ is

$$
\Lambda_{\Xi_{\alpha}, \mathbf{r}_{\alpha}}=\mathbb{Z}(q,-(q+1), 1) .
$$


Let $\lambda=\left(\lambda_{1}, \lambda_{2}, \lambda_{3}\right)$ be an $L$-dominant character (i.e., $\left.\lambda_{1} \geq \lambda_{2}\right)$ and consider the $L$-representation $V_{I}(\lambda)$. We simply write $V$ for $V_{I}(\lambda)$ sometimes. Under the isomorphism

$$
\mathrm{GL}_{2} \times \mathbb{G}_{\mathrm{m}} \rightarrow L ;(A, z) \mapsto\left(\begin{array}{ll}
A & \\
& z
\end{array}\right),
$$

the representation $V$ corresponds to the representation

$$
\operatorname{det}_{\mathrm{GL}_{2}}^{\lambda_{2}} \otimes \operatorname{Sym}^{\lambda_{1}-\lambda_{2}}\left(\operatorname{Std}_{\mathrm{GL}_{2}}\right) \otimes \xi_{\lambda_{3}},
$$

where $\xi_{r}$ is the character of $\mathrm{GL}_{2} \times \mathbb{G}_{\mathrm{m}}$ given by $(A, z) \mapsto z^{r}$. Hence, $V$ is a representation of dimension $\lambda_{1}-\lambda_{2}+1$ and it has weights

$$
v_{i}:=\left(\lambda_{1}-i, \lambda_{2}+i, \lambda_{3}\right), \quad 0 \leq i \leq \lambda_{1}-\lambda_{2} .
$$

Note that the difference $v_{i}-v_{i^{\prime}}$ of two weights is never in $\Lambda_{\Xi_{\alpha}, \mathbf{r}_{\alpha}}$ unless $i=i^{\prime}$. Therefore, $V_{[v]}=V_{v}$ for all $v \in \mathbb{Z}^{3}$. One deduces

$$
V^{L_{\varphi}}=\bigoplus_{\substack{q|i, q+1| \lambda_{2}+i \\ q^{2}-1 \mid \lambda_{1}-i-q \lambda_{3}}} V_{v_{i}}
$$

It remains to determine $\mathrm{Fil}_{\delta_{\alpha}}^{\Xi_{\alpha}, \mathbf{a}_{\alpha}, \mathbf{r}_{\alpha}} V_{v}$, which is either 0 or $V_{v}$. We parametrise elements $[\mathbf{j}] \in \mathbb{Z}^{2} /\left(\mathbb{Z}^{2}\right)_{\mathbf{r}_{\alpha}}$ by classes $[(0, j)]$ with $j \in \mathbb{Z}$. Then, an element $\mathbf{j} \in[\mathbf{j}]$ can be written as $(0, j)+j_{1}(1, q)$ with $j_{1} \in \mathbb{Z}$. Using this notation, we obtain

$$
\operatorname{Fil}_{\delta}^{\Xi, \mathbf{a}, \mathbf{r}} V_{v}=\bigcap_{j \in \mathbb{Z}} \bigcap_{\substack{\chi \in[v+j \sigma(\alpha)], j r_{\alpha, 2}>\delta_{\alpha}(\chi)}} \operatorname{Ker}\left(\sum_{j_{1} \in \mathbb{Z}} \operatorname{pr}_{\chi} \circ E_{-\alpha}^{\left(j_{1}\right)} \circ E_{\sigma(\alpha)}^{\left(j+q j_{1}\right)}: V_{v} \rightarrow V_{\chi}\right)
$$

because $(-1)^{j_{1}}(-1)^{j+q j_{1}}=(-1)^{j} \in k$. We have $E_{-\alpha}^{\left(j_{1}\right)}=0$ unless $j_{1}=0$ because $\alpha \in \Delta^{P}$ and $V$ is trivial on $R_{\mathrm{u}}(P)$. Hence, in the sum appearing in the above formula, only the case $j_{1}=0$ contributes. Furthermore, $E_{\sigma(\alpha)}^{(j)}\left(V_{v}\right) \subset V_{v+j \sigma(\alpha)}$. Hence, we have

$$
\mathrm{Fil}_{\delta}^{\Xi, \mathbf{a}, \mathbf{r}} V_{v}=\bigcap_{j>q\left\langle v, \delta_{\alpha}\right\rangle} \operatorname{Ker}\left(E_{e_{1}-e_{2}}^{(j)}: V_{v} \rightarrow V_{v+j\left(e_{1}-e_{2}\right)}\right) .
$$

Take $v=v_{i}$ for some $0 \leq i \leq \lambda_{1}-\lambda_{2}$. We deduce Fil ${ }_{\delta_{\alpha}}^{\Xi_{\alpha}, \mathbf{a}_{\alpha}, \mathbf{r}_{\alpha}} V_{v_{i}}=V_{v_{i}}$ if and only if for all $j \geq 0$ such that $j>q\left\langle v_{i}, \delta_{\alpha}\right\rangle$, one has $E_{e_{1}-e_{2}}^{(j)}\left(V_{v_{i}}\right)=0$. Computing explicitly the representation $V$, one sees that this space is zero if and only if the binomial coefficient $\left(\begin{array}{l}i \\ j\end{array}\right)$ is divisible by $p$. In particular, it is never zero for $j=i$. We deduce that

$$
\mathrm{Fil}_{\delta_{\alpha}}^{\Xi_{\alpha}, \mathbf{a}_{\alpha}, \mathbf{r}_{\alpha}} V_{v_{i}}=V_{v_{i}} \Longleftrightarrow i \leq q\left\langle v_{i}, \delta_{\alpha}\right\rangle .
$$

Furthermore, we find

$$
\left\langle v_{i}, \delta_{\alpha}\right\rangle=\frac{i(2 q-1)}{q^{2}-1}+\frac{1}{q^{2}-1}\left(-q \lambda_{1}+(q-1) \lambda_{2}+\lambda_{3}\right) .
$$

For $\lambda=\left(\lambda_{1}, \lambda_{2}, \lambda_{3}\right) \in X_{+, I}^{*}(T)$, we put

$$
F(\lambda)=\frac{q}{q^{2}-q+1}\left(q \lambda_{1}-(q-1) \lambda_{2}-\lambda_{3}\right) .
$$

We deduce the following. 
Proposition 6.3.2. We have

$$
H^{0}\left(G-\mathrm{Zip}^{\mu}, \nu_{I}(\lambda)\right)=\bigoplus_{\substack{q|i, q+1| \lambda_{2}+i, q^{2}-1 \mid \lambda_{1}-i-q \lambda_{3}, i \geq F(\lambda)}} V_{I}(\lambda)_{v_{i}}
$$

(1) For example, take $\lambda=(1+q, 1, q)$. Then one sees that $V_{I}(\lambda)^{L_{\varphi}}=V_{I}(\lambda)_{v_{q}}$, where $v_{q}=(1,1+q, q)$. One finds $F(\lambda)=q$; hence, $H^{0}\left(G-\operatorname{Zip}^{\mu}, \nu_{I}(\lambda)\right)=V_{I}(\lambda)_{v_{q}}$.

(2) Similarly, take $\lambda=(1,0, q)$. Then we find $V_{I}(\lambda)^{L_{\varphi}}=V_{I}(\lambda)_{v_{0}}$, where $v_{0}=\lambda=(1,0, q)$. We have $F(\lambda)=0$; hence, again $H^{0}\left(G-\operatorname{Zip}^{\mu}, V_{I}(\lambda)\right)=V_{I}(\lambda)_{v_{0}}$.

(3) Take $\lambda=\left(q+1, q+1, q^{2}+q\right)$. Then $V_{I}(\lambda)$ is a 1-dimensional representation of $L$ (i.e., a character), and $V_{I}(\lambda)^{L_{\varphi}}=V_{I}(\lambda)$. Because $F(\lambda)=-\frac{q\left(q^{2}-1\right)}{q^{2}-q+1}<0$, we have $H^{0}\left(G\right.$-Zip $\left.{ }^{\mu}, V_{I}(\lambda)\right)=V_{I}(\lambda)$. It is spanned by the $\mu$-ordinary (nonclassical) Hasse invariant $H$ given by Lemma 6.3.1, also constructed in [GN17] and [KW18].

Recall the cone $C_{\text {zip }} \subset X_{+, I}^{*}(T)$ studied in [Kos19], [GK18], defined as the set of $\lambda \in X^{*}(T)$ such that $H^{0}\left(G\right.$-Zip $\left.{ }^{\mu}, V_{I}(\lambda)\right) \neq 0$. In this example, we deduce that it is the set of $\lambda \in X_{+, I}^{*}(T)$ such that there exists $0 \leq i \leq \lambda_{1}-\lambda_{2}$ satisfying the four conditions listed below the direct sum sign of (6.3.1). For a cone $C \subset X^{*}(T)$, write $\langle C\rangle$ for the saturated cone of $C$; that is, the set of $\lambda \in X^{*}(T)$ such that $N \lambda$ lies in $C$ for some positive integer $N$.

Corollary 6.3.3. We have

$$
\left\langle C_{\text {zip }}\right\rangle=\left\{\left(\lambda_{1}, \lambda_{2}, \lambda_{3}\right) \in \mathbb{Z}^{3} \mid \lambda_{1} \geq \lambda_{2},(q-1) \lambda_{1}+\lambda_{2}-q \lambda_{3} \leq 0\right\} .
$$

Proof. Assume that $\lambda \in C_{\text {zip }}$. Then, in particular, $\lambda_{1}-\lambda_{2} \geq F(\lambda)$, which amounts to $(q-1) \lambda_{1}+\lambda_{2}-q \lambda_{3} \leq$ 0 . Conversely, assume that $\lambda \in X_{+, I}^{*}(T)$ satisfies $\lambda_{1}-\lambda_{2} \geq F(\lambda)$. Then, after changing $\lambda$ to $q\left(q^{2}-1\right) \lambda$, we find that $i=\lambda_{1}-\lambda_{2}$ satisfies the four conditions below the direct sum sign of (6.3.1); hence, $\lambda \in\left\langle C_{\text {zip }}\right\rangle$. This terminates the proof.

Remark 6.3.4. The two sections of weight $(1+q, 1, q)$ and $(1,0, q)$ given in (1) and (2) are partial Hasse invariants (viewing them as a section of the stack of zip flags $G$-ZipFlag ${ }^{\mu}$, their vanishing locus is a single flag stratum; see [Kos19, §1.3] for details). Their weights generate the cone $\left\langle C_{\text {Sbt }}\right\rangle$ defined in [Kos19, Definition 1.7.1]. The cone $\left\langle C_{\text {zip }}\right\rangle$ is not spanned by these weights because $G$ does not satisfy the equivalent conditions of [Kos19, Lemma 2.3.1]. We also refer to [GIK21] for a general study of the cone $C_{\text {zip }}$ as well as related results.

Acknowledgements. The authors thank the referee for helpful comments and suggestions. This work was supported by JSPS KAKENHI Grant Numbers 18F18311 and 18H01109.

Conflict of Interest: None.

\section{References}

[ABD+66] M. Artin, J. E. Bertin, M. Demazure, P. Gabriel, A. Grothendieck, M. Raynaud and J.-P. Serre, SGA3: Schémas en groupes [Group schemes], Vol. 1963/64 (Institut des Hautes Études Scientifiques, Paris, 1965/1966).

[Del79] P. Deligne, 'Variétés de Shimura: Interprétation modulaire, et techniques de construction de modèles canoniques' [Shimura varieties: Moduli interpretation and techniques of construction of canonical models], in Automorphic Forms, Representations and L-functions, Part 2, ed. by A. Borel and W. Casselman, Vol. 33 of Proc. Symp. Pure Math. (American Mathematical Society, Providence, RI, 1979), 247-289.

[Don85] S. Donkin, Rational Representations of Algebraic Groups: Tensor Products and Filtration, Vol. 1140 of Lecture Notes in Mathematics (Springer, Berlin, 1985).

[GIK21] W. Goldring, N. Imai and J.-S. Koskivirta, 'Weights of mod $p$ automorphic forms and partial Hasse invariants', Preprint, 2021. 
[GK18] W. Goldring and J.-S. Koskivirta, 'Automorphic vector bundles with global sections on G-Zip ${ }^{Z_{\text {-schemes', }}}$, Compositio Math. 154 (2018), 2586-2605.

[GK19a] W. Goldring and J.-S. Koskivirta, 'Strata Hasse invariants, Hecke algebras and Galois representations', Invent. Math. 217(3) (2019), 887-984.

[GK19b] W. Goldring and J.-S. Koskivirta, 'Zip stratifications of flag spaces and functoriality', IMRN (2019) (12) (2019), 3646-3682.

[GN17] W. Goldring and M.-H. Nicole, 'The $\mu$-ordinary Hasse invariant of unitary Shimura varieties', J. Reine Angew. Math. 728 (2017), 137-151.

[IK21] N. Imai and J.-S. Koskivirta, 'Partial Hasse invariants for Shimura varieties of Hodge-type', Preprint, 2021.

[Jan03] J. Jantzen, Representations of Algebraic Groups, Vol. 107 of Math. Surveys and Monographs, 2nd ed. (American Mathematical Society, Providence, RI, 2003).

[Kim18] W. Kim, 'Rapoport-Zink uniformization of Shimura varieties', Forum Math. Sigma 6 (2018), e16.

[Kis10] M. Kisin, 'Integral models for Shimura varieties of abelian type', J. Amer. Math. Soc. 23(4) (2010), 967-1012.

[Kos18] J.-S. Koskivirta, Normalization of closed Ekedahl-Oort strata, Can. Math. Bull. 613 (2018), $572-587$.

[Kos19] J.-S. Koskivirta, 'Automorphic forms on the stack of G-zips', Results Math. 74 (2019), no. 3, Paper No. 91,52 pp.

[KW18] J.-S. Koskivirta and T. Wedhorn, 'Generalized $\mu$-ordinary Hasse invariants', J. Algebra 502 (2018), 98-119.

[Kot84] R. E. Kottwitz, 'Shimura varieties and twisted orbital integrals', Math. Ann. 269 (1984), 287-300.

[Mi190] J. Milne, ' Canonical models of (mixed) Shimura varieties and automorphic vector bundles', in Automorphic Forms, Shimura Varieties, and L-Functions, Vol. I, ed. by L. Clozel and J. Milne, Vol. 11 of Perspect. Math. (Academic Press, Boston, 1990), 283-414.

[Moo04] B. Moonen, 'Serre-Tate theory for moduli spaces of PEL-type', Ann. Sci. ENS 37(2) (2004), $223-269$.

[MW04] B. Moonen and T. Wedhorn, 'Discrete invariants of varieties in positive characteristic', IMRN 72 (2004), 38553903.

[PWZ11] R. Pink, T. Wedhorn and P. Ziegler, 'Algebraic zip data', Doc. Math. 16 (2011), 253-300.

[PWZ15] R. Pink, T. Wedhorn and P. Ziegler, ' F-zips with additional structure', Pacific J. Math. 274(1) (2015), $183-236$.

[SYZ19] X. Shen, C.-F. Yu and C. Zhang, 'EKOR strata for Shimura varieties with parahoric level structure', Preprint, 2019, arXiv: 1910.07785 .

[Spr98] T. Springer, Linear Algebraic Groups, Vol. 9 of Progress in Math., 2nd edn. (Birkhauser, Boston, MA, 1998).

[Urb14] E. Urban, 'Nearly overconvergent modular forms', in Iwasawa Theory, Vol. 7 of Contrib. Math. Comput. Sci. (Springer, Heidelberg, Germany, 2014), 401-441.

[Vas99] A. Vasiu, 'Integral canonical models of Shimura varieties of preabelian type', Asian J. Math. 3 (1999), 401-518.

[Wor13] D. Wortmann, 'The $\mu$-ordinary locus for Shimura varieties of Hodge type', Preprint, 2013, arXiv:1310.6444.

[XZ17] L. Xiao and X. Zhu, 'Cycles on Shimura varieties via geometric Satake', Preprint, 2017, arXiv:1707.05700.

[XZ19] L. Xiao and X. Zhu, 'On vector-valued twisted conjugation invariant functions on a group', in Representations of Reductive Groups, Vol. 101 of Proc. Sympos. Pure Math. (American Mathematical Society, Providence, RI, 2019), 361-425. With an appendix by Stephen Donkin.

[Zha18] C. Zhang, 'Ekedahl-Oort strata for good reductions of Shimura varieties of Hodge type', Can. J. Math. 70(2) (2018), 451-480. 